
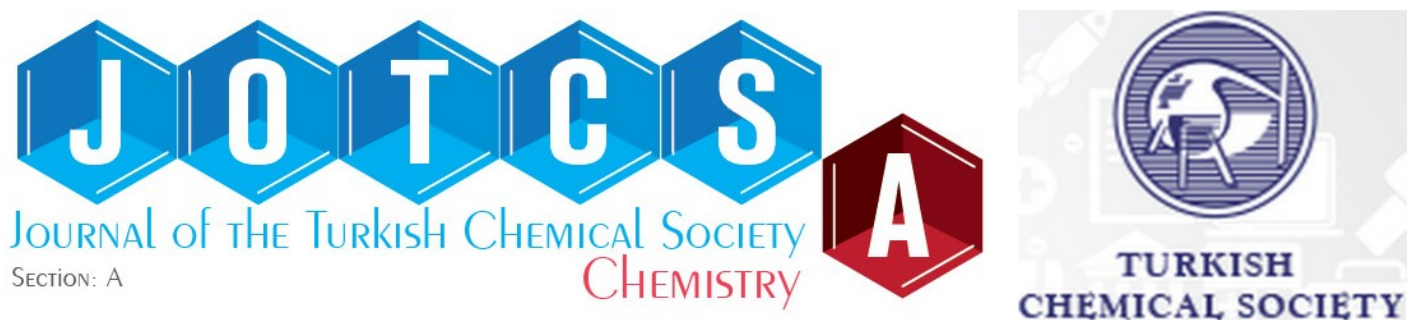

\title{
Can Soybean Lipoxygenases be Real Models for Mammalian Lipoxygenases? A Bioinformatics Approach
}

\author{
Sevilay CENGiz ŞAHIN* $\triangle$ iD, Levent CAVAS
}

${ }^{1}$ University of Pamukkale, Faculty of Arts and Sciences, Department of Molecular Biology and Genetics, 20070, Kınıklı Campus, DENIZLI-TURKEY.

2University of Dokuz Eylul, Faculty of Science, Department of Chemistry, Biochemistry Division, 35160, Kaynaklar Campus, IZMIR-TURKEY.

\begin{tabular}{|c|}
\hline 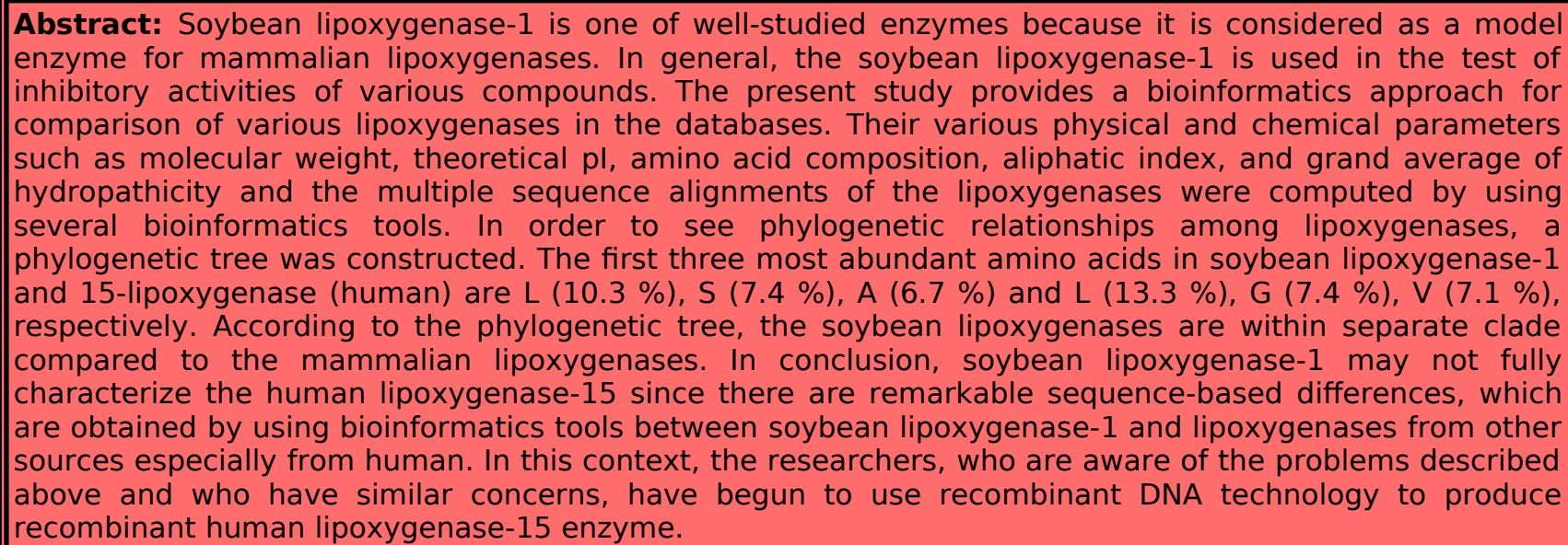 \\
\hline
\end{tabular}

Keywords: Bioinformatics, lipoxygenases, soybean lipoxygenase.

Submitted: August 25, 2020. Accepted: November 18, 2020.

Cite this: Cengiz Şahin S, Cavas L. Can Soybean Lipoxygenases be Real Models for Mammalian Lipoxygenases? JOTCSA. 2021;8(1):79-102.

DOI: https://doi.org/10.18596/jotcsa.785109.

*Corresponding author. E-mail: scengiz@pau.edu.tr, Tel: +902582963519.

\section{INTRODUCTION}

Lipoxygenases (EC 1.13.11.12, linoleate:oxygen, oxidoreductases, LOXs) which are a large protein family are widely distributed in animals, plants and fungi. These enzymes are also found in cyanobacteria, although they are rarely found in other prokaryotes $(1,2)$. Its general structure contained non-heme, non-sulfur iron atom which is responsible for enzymic activity. LOXs belong to dioxygenase family and they catalyze the oxidation of the polyunsaturated fatty acids (PUFA) such as linoleate, linolenate and arachidonate to form hydroperoxides utilizing $\mathrm{Fe}^{+2} / \mathrm{Fe}^{+3}$ redox potential and molecular oxygen $(3,4)$. Mammalian LOXs are named as 5-LOX, 8-LOX, 11-LOX 12-LOX and 15LOX, whereas it is 9-LOX and 13-LOX in plants based on the peroxidation position of PUFAs (5-7). Boyington et al (8) identified the first crystalline structure of LOXs from soybean that is previously named as soybean LOX-1. Although this enzyme was firstly characterized in 1947, the comprehensive analyses of soybean LOX-1 biochemistry were conducted following the structural identification in 1993 (9). Among LOXs, soybean LOX-1 is one of the well-studied LOX enzymes due to its ease of purification and activation stability $(10,11)$ and also it has been considered as a model LOX for 
mammalian LOXs, especially for human due to the insufficient purified human LOXs (5,8,12-19). According to the results of X-ray crystalline analysis, soybean LOX-1 has two domains: a smaller $\beta$-barrel domain and a much larger $\alpha$-helix domain included the catalytic iron and substrate binding cavity $(8,20,21)$. LOX enzymes generally have a highly conserved Fe-coordination sphere within the $\alpha$-helix structure. Three His and one lle ligands in the active site are also conserved in all lipoxygenases except from rat leukocyte 5-LOX (22,23). Minor et al (24) reported that the active site had two additional ligands as a water and Asn694 which can be responsible for the coordination flexibility of the active site. Although the highly conserved region in catalytic site motivate researchers to use soybean LOX-1 as a model for 15-LOX (human), it should be kept in mind that minor changes in conserved structure may lead significant functional differences between related enzymes (2). For instance, the reaction mechanism of 15-LOX (human) is considered to be similar to soybean LOX-1, but the $\mathrm{k}_{\text {cat }}$ value of 15-LOX (human) is approximately 45fold lower than that of soybean LOX-1. The decrease in the $k_{\text {cat }}$ value might have been caused by the change in redox potential of iron as a result of ligation difference (23).

LOXs have stimulated interests of many researchers so far because of the roles of these enzymes in many diseases such as bronchial asthma, allergic rhinitis, inflammatory bowel, rheumatoid arthritis, osteoporosis, stroke, cardiovascular diseases, Alzheimer's disease, and cancer. Moreover, LOX products, leukotrienes, and lipoxins, have a part in significant metabolic functions such as organelle degradation (25), transcription regulation (26), and possibly tumor cell metastasis (27). Therefore, the inhibition of LOXs is considered an important target for the treatment of LOX-related diseases (5$7,16,28-35)$. There are some reports which show the results of LOX inhibition studies in the literature. The inhibitory effects of three Malian plant extracts with different polarities on soybean 15-LOX were determined by Maiga et al (14). The inhibitory potential of substituted benzoic acids which is widely found in plants on soybean 15-LOX was studied by Russell et al (17). In another report conducted by Bouriche et al (36), the effects of Cleome arabica leaf extract, rutin, and quercetin on soybean LOX were examined. Mahesha et al (16) investigated the inhibitory effect of genistein and daidzein compounds against soybean LOX. Plant polyphenols known as sinapic acid, caffeic acid, chlorogenic acid, ferulic acid, naringenin, and chrysin were evaluated as an inhibitor of lipoxygenase and chlorogenic acid was determined as the strongest inhibitor for soybean LOX with an $\mathrm{IC}_{50}$ values of $61.27 \mu \mathrm{mol} / \mathrm{L}$ (37). The inhibitory effect of sesamol, the phenolic degradation product of sesamolin, on lipoxygenase was determined in another investigation. It is reported that sesamol inhibited the soybean LOX-1 in a dose dependent manner with $\mathrm{IC}_{50}$ value of $51.84 \mu \mathrm{M}$ and the enzyme kinetic results indicated that the inhibition occurred in a competitive manner with the Ki value of $4.9 \mu \mathrm{M}$ (6). In above mentioned studies, soybean LOX has been used as a model enzyme for mammalian LOXs. On the other hand, the developments in bioinformatics and computational biology have provided a big contribution to understanding of proteins more comprehensively. Bioinformatics tools such as Clustal Omega $(38,39)$, some tools in expasy.ch $(40,41)$ such as PROTPARAM $(41)$ and also phylogenetic tree construction $(38,39)$ might help for a detailed comparison of LOXs.

This investigation aims at answering the following research questions:

- Is soybean LOX family phylogenetically close to mammalian LOX family?

- $\quad$ Are there any differences among various physical and chemical parameters of LOXs; such as molecular weight, theoretical pl, amino acid composition, aliphatic index and grand average of hydropathicity (GRAVY)?

- Is it reliable enough to use soybean LOXs in inhibition studies as a model for mammalian LOXs?

\section{IN SILICO EXPERIMENTAL SECTION}

The sequences and related biochemical reactions of the LOXs studied were retrieved from proteomics server of the Swiss Institute of Bioinformatics ExPASy (Expert Protein Analysis System) (40). LOXs analyzed and their corresponding codes were presented in Tables 1 and 2 . Their various physical and chemical parameters such as molecular weight, theoretical pl, amino acid composition, aliphatic index, and GRAVY were computed by using PROTPARAM tool of Expasy.ch $(40,41)$. These parameters were also shown in Tables 3 and 4 . The multiple sequence alignments of the LOXs were also computed by using Clustal Omega $(38,39)$. In order to understand the evolutionary relationship between the LOXs studied, a phylogenetic analysis was carried out. Phylogenetic tree was constructed via phylogenetic tool at Clustal Omega web tool $(38,39)$. In order to achieve the secondary structure prediction of soybean LOX-1 and 15-LOX (human), the PSIPRED Protein Structure Prediction Server was used (42). Table 7 represents the conserved regions of the aligned sequences. 
Table 1. Coding used for analyzed lipoxygenases and related database information

\begin{tabular}{|c|c|c|c|c|c|}
\hline Code & Protein Name & Abbreviation & Gene Name & $\begin{array}{l}\text { Primary Accession Number } \\
\text { in Swiss-Prot }\end{array}$ & Species \\
\hline$\overline{\mathbf{1}}$ & Polyunsaturated fatty acid 5-lipoxygenase & $5-\mathrm{LO}$ & ALOX5 & P09917 & Homo sapiens (Human) \\
\hline 2 & Arachidonate 12 -lipoxygenase, 12R-type & 12R-LOX & ALOX12B & 075342 & Homo sapiens (Human) \\
\hline 3 & Polyunsaturated fatty acid 5-lipoxygenase & 5-LO & Alox5 & P12527 & Rattus norvegicus (Rat) \\
\hline 4 & Arachidonate 12 -lipoxygenase, 12R-type & 12R-LOX & Alox $12 b$ & 070582 & Mus musculus (Mouse) \\
\hline 5 & Polyunsaturated fatty acid lipoxygenase ALOX12 & 12S-LOX & ALOX12 & P18054 & Homo sapiens (Human) \\
\hline 6 & Seed linoleate 13S-lipoxygenase-1 & L-1 & LOX1.1 & P08170 & Glycine max (Soybean) \\
\hline 7 & Seed linoleate 9S-lipoxygenase-3 & L-3 & LOX1.3 & P09186 & Glycine max (Soybean) \\
\hline 8 & Polyunsaturated fatty acid lipoxygenase ALOX15 & 15-LOX & ALOX15 & P16050 & Homo sapiens (Human) \\
\hline 9 & Polyunsaturated fatty acid 5-lipoxygenase & $5-\mathrm{LO}$ & Alox5 & P48999 & Mus musculus (Mouse) \\
\hline 10 & Arachidonate 12-lipoxygenase, 12R-type & 12R-LOX & Alox12b & Q2KMM4 & Rattus norvegicus (Rat) \\
\hline 11 & Polyunsaturated fatty acid 5-lipoxygenase & 5-LO & ALOX5 & P51399 & Mesocricetus auratus (Golden hamster) \\
\hline 12 & Polyunsaturated fatty acid lipoxygenase ALOX15 & 15-LOX & ALOX15 & P12530 & Oryctolagus cuniculus (Rabbit) \\
\hline 13 & Polyunsaturated fatty acid lipoxygenase ALOX15B & 15-LOX-B & ALOX15B & 015296 & Homo sapiens (Human) \\
\hline 14 & Polyunsaturated fatty acid lipoxygenase ALOX8 & 8-LOX & Alox8 & 035936 & Mus musculus (Mouse) \\
\hline 15 & $\begin{array}{l}\text { Polyunsaturated fatty acid (12S)/(13S)- } \\
\text { lipoxygenase, epidermal-type }\end{array}$ & 12-LOX-e & Alox12e & P55249 & Mus musculus (Mouse) \\
\hline 16 & Polyunsaturated fatty acid lipoxygenase ALOX12 & 12S-LOX & Alox12 & P39655 & Mus musculus (Mouse) \\
\hline 17 & Hydroperoxide isomerase ALOXE3 & e-LOX-3 & ALOXE3 & Q9BYJ1 & Homo sapiens (Human) \\
\hline 18 & Seed linoleate 9S-lipoxygenase & - & LOX1.4 & P24095 & Glycine max (Soybean) \\
\hline 19 & Seed linoleate 9S-lipoxygenase-2 & L-2 & LOX1.2 & P09439 & Glycine max (Soybean) \\
\hline 20 & Polyunsaturated fatty acid lipoxygenase ALOX15 & 15-LOX & Alox15 & P39654 & Mus musculus (Mouse) \\
\hline 21 & Polyunsaturated fatty acid lipoxygenase ALOX15 & 15-LOX & Alox15 & Q02759 & Rattus norvegicus (Rat) \\
\hline 22 & Polyunsaturated fatty acid lipoxygenase ALOX15 & 15-LOX & ALOX15 & P16469 & Sus scrofa (Pig) \\
\hline 23 & Linoleate 9S-lipoxygenase- 4 & $\mathrm{~L}-4$ & LOX1.5 & P38417 & Glycine max (Soybean) \\
\hline 24 & Polyunsaturated fatty acid lipoxygenase ALOX15B & 15-LOX-B & Alox15b & Q8K4F2 & Rattus norvegicus (Rat) \\
\hline 25 & Polyunsaturated fatty acid lipoxygenase ALOX15 & 15-LOX & ALOX15 & P27479 & Bos taurus (Bovine) \\
\hline 26 & Hydroperoxide isomerase ALOXE3 & e-LOX-3 & Aloxe3 & Q9WV07 & Mus musculus (Mouse) \\
\hline
\end{tabular}




\section{RESULTS AND DISCUSSION}

The functions and the catalytic activities of LOXs studied were summarized in Table 2 . When the Table 2 is evaluated, it is very clear that LOXs from plants and vertebrates do not reveal completely same biochemical catalytic reactions.

Some chemical and physicochemical properties of LOXs studied such as number of amino acids, molecular weight (Da), theoretical pl, number of negatively and positively charged amino acids, aliphatic index and GRAVY were shown in Table 3. The molecular weight of soybean LOX-1 is higher than that of 15-LOX (human). The length of sequences for soybean LOXs are generally longer than those for mammalian ones. Although the soybean LOX have more amino acid residues in its general structure than mammalian LOX, approximately 200 amino acids, there is a little difference between the pl values of these species. The solubility characteristics of the proteins can be expressed via GRAVY index as the positive and negative values of it shows hydrophobic and hydrophilic characters, respectively (43). As it is seen in the Table 3, the value of GRAVY for soybean LOXs is more negative than those of mammalians. So it can be said that soybean LOXs are more soluble in polar solvents. The minimum and maximum theoretical pl values were observed as 5.51 and 8.31 for polyunsaturated fatty acid 5lipoxygenase and polyunsaturated fatty acid (12S)/(13S)-lipoxygenase, epidermal-type, respectively. It is well known that the amino acids in active site are responsible for the enzymic activity. Although these differences mentioned above exhibit the difference of evolutionary origins of these enzymes, the similarities in active site composition reveal convergent evolution. As can be seen from Table 3, the amino acid residues in active sites of all LOXs studied are very similar. Mogul et al (44) suggested that the active site of soybean LOX-1 and 15-LOX (human) is slightly different according to the results of inhibition studies conducted with alkenyl-sulfate substrates. It was reported that alkenyl-sulfates are not inhibitors for soybean LOX-1. They cannot inhibit
15-LOX (human) at low concentrations, either. But 15-LOX (human) is irreversibly inhibited by alkenylsulfates at high concentrations. Moreover, different enzymic activities were reported for LOXs from different origins in the scientific literature. For example, the activity of soybean LOX-1 was reported as $280 \mu \mathrm{mol} / \mathrm{min} . \mathrm{mg}$ (45). On the other hand, the enzymic activity of 15-LOX from Pseudomonas aeruginosa (46) was reported to be $747130.2 \mathrm{IU} / \mathrm{mg}$ protein with linoleic acid. Therefore, there might be another parameter such as water or different ligand-substrate interactions except from the amino acid residues in active sites which can help researchers to a better understanding about the activity variations among LOXs. Iron atom in the active site of LOXs is essential for the enzymatic activity and it is believed that the micro environment of this atom is crucial for the reaction $(23,31)$. Iron in the active site has six coordinates which were identified as His499, His504, His690, Asn694, the terminal carboxylate of lle839 and an additional water ligand $(8,24)$. The enzymatic activity (or the rate of the reaction) is affected by the various changes in this environment. For example, the Asn694 residue is converted His in mammalian LOX (15-LOX (rabbit)). As the His is a stronger ligand than Asn, the rate of the reaction is decreased as a result of the changes in the reduction potential of iron ion and the pKa value $(23,31)$.

The amino acid compositions of LOXs are shown in Table 4. The first three most abundant amino acids in soybean LOX-1 and LOX-15 (human) are L (10.3 $\%), S(7.4 \%), A(6.7 \%)$ and L (13.3\%), G (7.4 \%), $\mathrm{V}(7.1 \%)$, respectively. It is known that the number of amino acids between plant and mammalian LOXs are significantly different. Mammalian LOXs have nearly 662-711 amino acids (in the range of 75-81 kDa for molecular weight) while plant LOXs have 838-923 amino acids (in the range of 94-103 $\mathrm{kDa}$ for molecular weight). It is very surprising that despite the difference between the amino acid number of plant and mammalian LOXs, the topologies and the catalytic site are highly conserved in LOX family $(21,47)$. 
Polyunsaturated fatty acid 5-lipoxygenase (Human)

\section{Arachidonate 12-lipoxygenase, 12R type} (Human)

Polyunsaturated fatty acid 5-lipoxygenase (Rat)

Arachidonate 12-lipoxygenase, 12R-type (Mouse)

Polyunsaturated fatty acid lipoxygenase ALOX12 (Human)

Seed linoleate 13S-lipoxygenase-1 (Soybean)

Seed linoleate 9S-lipoxygenase-3 (Soybean)

Polyunsaturated fatty acid lipoxygenase ALOX15 (Human)
It catalyzes the oxygenation of arachidonic acid to 5hydroperoxyicosatetraenoic acid (5-HPETE) followed by the dehydration of the hydroperoxide into an epoxide, 5,6oxidoicosatetraenoic acid (LTA4), thereby participates in the first step in leukotriene biosynthesis and in the inflammatory processes.

It catalyzes the regio and stereo-specific incorporation of a single molecule of dioxygen into free and esterified polyunsaturated fatty acids generating lipid hydroperoxides that can be further reduced to the corresponding hydroxy species.

It catalyzes the oxygenation of arachidonic acid to 5-

hydroperoxyicosatetraenoic acid (5-HPETE) followed by the dehydration of the hydroperoxide into an epoxide, 5,6oxidoicosatetraenoic acid (LTA4), thereby participates in the first step in leukotriene biosynthesis and in the inflammatory processes.

It catalyzes the regio and stereo-specific incorporation of a single molecule of dioxygen into free and esterified polyunsaturated fatty acids generating lipid hydroperoxides that can be further reduced to the corresponding hydroxy species. Does not convert arachidonic acid to (12R)-hydroperoxyeicosatetraenoic acid/(12R)-HPETE.

It catalyzes the regio and stereo-specific incorporation of a single molecule of dioxygen into free and esterified polyunsaturated fatty acids generating lipid hydroperoxides that can be further reduced to the corresponding hydroxy species. Mainly converts arachidonic acid to (12S)-hydroperoxyeicosatetraenoic acid/(12S)-HPETE but can also metabolize linoleic acid. Plant lipoxygenase may be involved in a number of diverse aspects of plant physiology including growth and development pest resistance, and senescence or responses to wounding. With linoleate as substrate, L-1 shows a preference for carbon 13 as the site for hydroperoxidation (in contrast to L-2 and L-3, which utilize either carbon 9 or 13).

Plant lipoxygenase may be involved in a number of diverse aspects of plant physiology including growth and development, pest resistance, and senescence or responses to wounding. It catalyzes the hydroperoxidation of lipids containing a cis, cis-1,4pentadiene structure.

It catalyzes the stereo-specific peroxidation of free and esterified polyunsaturated fatty acids generating a spectrum of bioactive lipid mediators. It converts arachidonic acid into 12hydroperoxyeicosatetraenoic acid/12-HPETE and 15hydroperoxyeicosatetraenoic acid/15-HPETE. Also converts linoleic acid to 13-hydroperoxyoctadecadienoic acid. May also act on (12S)-hydroperoxyeicosatetraenoic acid/(12S)-HPETE to produce hepoxilin A3.
$(5 \mathrm{Z}, 8 \mathrm{Z}, 11 \mathrm{Z}, 14 \mathrm{Z})$-eicosatetraenoate $+\mathrm{O}_{2}=$ $\mathrm{H}_{2} \mathrm{O}$ + leukotriene $\mathrm{A}_{4}$

5Z,8Z,11Z,14Z)-eicosatetraenoate $+\mathrm{O}_{2}=$ (12R)-hydroperoxy-(5Z,8Z,10E,14Z)eicosatetraenoate

$(5 Z, 8 Z, 11 Z, 14 Z)$-eicosatetraenoate $+\mathrm{O}_{2}=$ $\mathrm{H}_{2} \mathrm{O}+$ leukotriene $\mathrm{A}_{4}$

1-O-methyl-(5Z,8Z,11Z,14Z)eicosatetraenoate $+\mathrm{O}_{2}=1$-O-methyl $(5 Z, 8 Z, 10 E, 12 R, 14 Z)$

hydroperoxyiecosatetraenoate

$(5 Z, 8 Z, 11 Z, 14 Z)$-eicosatetraenoate $+\mathrm{O}_{2}=$ (12S)-hydroperoxy-(5Z,8Z,10E,14Z) eicosatetraenoate

9Z,12Z)-octadecadienoate $+\mathrm{O}_{2}=(13 S)$ hydroperoxy-(9Z,11E)-octadecadienoate

$(9 \mathrm{Z}, 12 \mathrm{Z})$-octadecadienoate $+\mathrm{O}_{2}=(9 \mathrm{~S})$ hydroperoxy-(10E,12Z)-octadecadienoate

$(5 Z, 8 Z, 11 Z, 14 Z)$-eicosatetraenoate $+\mathrm{O}_{2}=(1$ $2 S)$-hydroperoxy- $(5 Z, 8 Z, 10 E, 14 Z)$ eicosatetraenoate 
Polyunsaturated fatty acid 5-lipoxygenase (Mouse)

Arachidonate 12-lipoxygenase, 12R-type (Rat)

Polyunsaturated fatty acid 5-lipoxygenase (Golden hamster)

Polyunsaturated fatty acid lipoxygenase ALOX15 (Rabbit)

Polyunsaturated fatty acid lipoxygenase ALOX15B (Human)

\section{Polyunsaturated fatty acid lipoxygenase ALOX8} (Mouse)

Polyunsaturated fatty acid (12S)/(13S)lipoxygenase, epidermal-type (Mouse)

Polyunsaturated fatty acid lipoxygenase ALOX12 (Mouse)

Hydroperoxide isomerase ALOXE3 (Human)
It catalyzes the oxygenation of arachidonic acid to 5hydroperoxyicosatetraenoic acid (5-HPETE) followed by the dehydration of the hydroperoxide into an epoxide, 5,6oxidoicosatetraenoic acid (LTA4), thereby participates in the first step in leukotriene biosynthesis and in the inflammatory processes.

It catalyzes the regio and stereo-specific incorporation of a single molecule of dioxygen into free and esterified polyunsaturated fatty acids generating lipid hydroperoxides that can be further reduced to the corresponding hydroxy species.

It catalyzes the oxygenation of arachidonic acid to 5hydroperoxyicosatetraenoic acid (5-HPETE) followed by the dehydration of the hydroperoxide into an epoxide, 5,6-

oxidoicosatetraenoic acid (LTA4), thereby participates in the first step in leukotriene biosynthesis and in the inflammatory processes.

It catalyzes the stereo-specific peroxidation of free and esterified polyunsaturated fatty acids generating a spectrum of bioactive lipid mediators. It converts arachidonic acid into 12-

droperoxyeicosatetraenoic acid/15-HPETE Also converts linoleic acid to 13-hydroperoxyoctadecadienoic acid. May also linole (12S)-hydroperoxyeicosatetraenoic acid/(12S)-HPETE to produce hepoxilin A3.

produce hepoxilin $A 3$.
It catalyzes the stereo-specific peroxidation of free and esterified It catalyzes the stereo-specific peroxidation of free and esterified polyunsaturated fatty acids (PUFAs) generating a spectrum of
bioactive lipid mediators. It converts arachidonic acid to 15Sbioactive lipid mediators. It converts arachidonic acid to 15S-
hydroperoxyeicosatetraenoic acid/(15S)-HPETE. Also acts on linoleic acid to produce 13-hydroxyoctadecadienoic acid/13HPODE.

It catalyzes the stereo-specific peroxidation of free and esterified polyunsaturated fatty acids (PUFAs) generating a spectrum of bioactive lipid mediators. It catalyzes the peroxidation of arachidonate and linoleate into (8S)-HPETE and (9S)-HPODE respectively.

It catalyzes the regio and stereo-specific incorporation of a single molecule of dioxygen into free and esterified polyunsaturated fatty acids generating lipid hydroperoxides that can be further fatty acids generating lipid hydroperoxides that can be furt
reduced to the corresponding hydroxy species.

reduced to the corresponding hydroxy species.
yzes the regio and stereo-specific incorporation of a single It catalyzes the regio and stereo-specific incorporation of a sing molecule of dioxygen into free and esterified polyunsaturated fatty acids generating lipid hydroperoxides that can be further arachidonic acid to (12S)-hydroperoxyeicosatetraenoic acid/(12S)-HPETE but can also metabolize linoleic acid. In contrast does not react towards methyl esters of linoleic and arachidonic acids.

Non-heme iron-containing lipoxygenase which is atypical in that Non-heme iron-containing lipoxygenase which is atypical in that it displays a prominent hydroperoxide isomerase activity and activity catalyzes the isomerization of hydroperoxides, derived
$(5 Z, 8 Z, 11 Z, 14 Z)$-eicosatetraenoate $+\mathrm{O}_{2}=$ (5S)-hydroperoxy-(6E,8Z,11Z,14Z)eicosatetraenoate

$(5 Z, 8 Z, 11 Z, 14 Z)$-eicosatetraenoate $+\mathrm{O}_{2}=(1$ $2 R)$-hydroperoxy-(5Z,8Z,10E,14Z) eicosatetraenoate

$(5 Z, 8 Z, 11 Z, 14 Z)$-eicosatetraenoate $+\mathrm{O}_{2}=$ $\mathrm{H}_{2} \mathrm{O}+$ leukotriene $\mathrm{A}_{4}$

$(5 Z, 8 Z, 11 Z, 14 Z)$-eicosatetraenoate $+\mathrm{O}_{2}=(1$ $2 S)$-hydroperoxy-(5Z,8Z,10E,14Z)eicosatetraenoate

$(5 Z, 8 Z, 11 Z, 14 Z)$-eicosatetraenoate $+\mathrm{O}_{2}=(1$ $5 S)$-hydroperoxy-(5Z,8Z,11Z,13E)eicosatetraenoate

$(9 \mathrm{Z}, 12 \mathrm{Z})$-octadecadienoate $+\mathrm{O}_{2}=(9 \mathrm{~S})$ hydroperoxy-(10E,12Z)-octadecadienoate

$(5 Z, 8 Z, 11 Z, 14 Z)$-eicosatetraenoate $+\mathrm{O}_{2}=(1$ $2 S)$-hydroperoxy- $(5 Z, 8 Z, 10 E, 14 Z)$ eicosatetraenoate

$(5 Z, 8 Z, 11 Z, 14 Z)$-eicosatetraenoate $+\mathrm{O}_{2}=(1$ $2 S)$-hydroperoxy-(5Z,8Z,10E,14Z)eicosatetraenoate

a hydroperoxyeicosatetraenoate $=$ a hydroxyepoxy-eicosatetraenoate 
Seed linoleate 9S-lipoxygenase (Soybean)

Seed linoleate 9S-lipoxygenase-2 (Soybean)

Polyunsaturated fatty acid lipoxygenase ALOX15 (Mouse)

Polyunsaturated fatty acid lipoxygenase ALOX15 (Rat)

Polyunsaturated fatty acid lipoxygenase ALOX15 (Pig)

Linoleate 9S-lipoxygenase-4 (Soybean)

Polyunsaturated fatty acid lipoxygenase ALOX15B (Rat) from arachidonic and linoleic acid by ALOX12B, into hepoxilintype epoxyalcohols and ketones. In presence of oxygen,

oxygenates polyunsaturated fatty acids, including arachidonic acid, to produce fatty acid hydroperoxides.

Plant lipoxygenase may be involved in a number of diverse aspects of plant physiology including growth and development, pest resistance, and senescence or responses to wounding. It catalyzes the hydroperoxidation of lipids containing a cis,cis-1,4pentadiene structure.

Plant lipoxygenase may be involved in a number of diverse aspects of plant physiology including growth and development aspects of plant physiology including growth and development,
pest resistance, and senescence or responses to wounding. It catalyzes the hydroperoxidation of lipids containing a cis,cis-1,4catalyzes the hydroperoxidation of lipids containing a cis,cis-1,4-
pentadiene structure. pentadiene structure.

It catalyzes the stereo-specific peroxidation of free and esterified polyunsaturated fatty acids generating a spectrum of bioactive

lipid mediators. It converts arachidonic acid into 12-

hydroperoxyeicosatetraenoic acid/12-HPETE and 15-

hydroperoxyeicosatetraenoic acid/15-HPETE. Also converts linoleic acid to 13-hydroperoxyoctadecadienoic acid. May also act on (12S)-hydroperoxyeicosatetraenoic acid/(12S)-HPETE to produce hepoxilin A3. Probably plays an important role in the immune and inflammatory responses.

It catalyzes the stereo-specific peroxidation of free and esterified polyunsaturated fatty acids generating a spectrum of bioactive

lipid mediators. It converts arachidonic acid into 12-

hydroperoxyeicosatetraenoic acid/12-HPETE and 15-

hydroperoxyeicosatetraenoic acid/15-HPETE. Also converts linoleic acid to 13-hydroperoxyoctadecadienoic acid. May also act on (12S)-hydroperoxyeicosatetraenoic acid/(12S)-HPETE to produce hepoxilin A3. Probably plays an important role in the immune and inflammatory responses.

It catalyzes the stereo-specific peroxidation of free and esterified polyunsaturated fatty acids generating a spectrum of bioactive

lipid mediators. It converts arachidonic acid into 12-

hydroperoxyeicosatetraenoic acid/12-HPETE and 15-

hydroperoxyeicosatetraenoic acid/15-HPETE. Also converts

linoleic acid to 13-hydroperoxyoctadecadienoic acid. May also act on (12S)-hydroperoxyeicosatetraenoic acid/(12S)-HPETE to produce hepoxilin A3. Probably plays an important role in the immune and inflammatory responses.

Plant lipoxygenase may be involved in a number of diverse aspects of plant physiology including growth and development, pest resistance, and senescence or responses to wounding. It catalyzes the hydroperoxidation of lipids containing a cis, cis-1,4pentadiene structure.

It catalyzes the stereo-specific peroxidation of free and esterified polyunsaturated fatty acids generating a spectrum of bioactive lipid mediators. It converts arachidonic acid to 15S-

hydroperoxyeicosatetraenoic acid/(15S)-HPETE. Also acts on linoleic acid to produce 13-hydroxyoctadecadienoic acid/13-
$(9 Z, 12 Z)$-octadecadienoate $+\mathrm{O}_{2}=(9 S)$ hydroperoxy-(10E,12Z)-octadecadienoate

$(9 Z, 12 Z)$-octadecadienoate $+\mathrm{O}_{2}=(9 S)$ hydroperoxy-(10E,12Z)-octadecadienoate

$(5 Z, 8 Z, 11 Z, 14 Z)$-eicosatetraenoate $+\mathrm{O}_{2}=(1$ $2 S)$-hydroperoxy- $(5 Z, 8 Z, 10 E, 14 Z)$ eicosatetraenoate

$(5 Z, 8 Z, 11 Z, 14 Z)$-eicosatetraenoate $+\mathrm{O}_{2}=(1$ $2 S)$-hydroperoxy-(5Z,8Z,10E,14Z)eicosatetraenoate

$(5 Z, 8 Z, 11 Z, 14 Z)$-eicosatetraenoate $+\mathrm{O}_{2}=(1$ $2 S)$-hydroperoxy-(5Z,8Z,10E,14Z)eicosatetraenoate

$(9 Z, 12 Z)$-octadecadienoate $+\mathrm{O}_{2}=(9 S)$ hydroperoxy-(10E,12Z)-octadecadienoate

$(5 Z, 8 Z, 11 Z, 14 Z)$-eicosatetraenoate $+\mathrm{O}_{2}=(1$ $5 S)$-hydroperoxy-(5Z,8Z,11Z,13E)eicosatetraenoate 
It catalyzes the stereo-specific peroxidation of free and esterified polyunsaturated fatty acids generating a spectrum of bioactive lipid mediators. It converts arachidonic acid into 12hydroperoxyeicosatetraenoic acid/12-HPETE and 15-

Polyunsaturated fatty acid lipoxygenase ALOX15 (Bovine)

Hydroperoxide isomerase ALOXE3 (Mouse) hydroperoxyeicosatetraenoic acid/15-HPETE. Also converts

linoleic acid to 13-hydroperoxyoctadecadienoic acid. May also act on (12S)-hydroperoxyeicosatetraenoic acid/(12S)-HPETE to produce hepoxilin A3. Probably plays an important role in the immune and inflammatory responses.

Non-heme iron-containing lipoxygenase which is atypical in that it displays a prominent hydroperoxide isomerase activity and rispla reduced lipoxygenases activity. The hydroperoxide isomerase activity catalyzes the isomerization of hydroperoxides, de from arachidonic and linoleic acid by ALOX12B, into hepoxilin type epoxyalcohols and ketones. In presence of oxygen,

oxygenates polyunsaturated fatty acids, including arachidonic
$(5 Z, 8 Z, 11 Z, 14 Z)$-eicosatetraenoate $+\mathrm{O}_{2}=(1$ $2 S)$-hydroperoxy-(5Z,8Z,10E, 14Z)eicosatetraenoate

a hydroperoxyeicosatetraenoate $=$ a hydroxyepoxy-eicosatetraenoate acid, to produce fatty acid hydroperoxides. 
Table 3. Protein characteristics of lipoxygenases analyzed.

\begin{tabular}{|c|c|c|c|c|c|c|c|c|c|c|c|c|c|c|c|c|}
\hline Proteins & 1 & 2 & 3 & 4 & 5 & 6 & 7 & 8 & 9 & 10 & 11 & 12 & 13 & 14 & 15 & 16 \\
\hline $\begin{array}{c}\text { Number of amino } \\
\text { acids }\end{array}$ & 674 & 701 & 673 & 701 & 663 & 839 & 857 & 662 & 674 & 701 & 673 & 663 & 676 & 677 & 662 & 663 \\
\hline $\begin{array}{l}\text { Molecular Weight } \\
\text { (Da) } \times 10^{3}\end{array}$ & 78.0 & 80.4 & 78.1 & 80.6 & 75.7 & 94.4 & 96.8 & 74.8 & 78.0 & 80.7 & 77.9 & 75.3 & 75.9 & 76.2 & 75.5 & 75.4 \\
\hline Theoretical pl & 5.51 & 7.57 & 5.85 & 6.25 & 5.82 & 5.96 & 6.26 & 6.14 & 5.78 & 6.43 & 5.81 & 6.15 & 5.73 & 6.25 & 8.31 & 5.79 \\
\hline $\begin{array}{c}\text { Number of negatively } \\
\text { charged residues } \\
\text { (Asp + Glu) }\end{array}$ & 92 & 75 & 91 & 77 & 82 & 101 & 106 & 80 & 89 & 76 & 89 & 75 & 73 & 75 & 78 & 79 \\
\hline $\begin{array}{c}\text { Number of positively } \\
\text { charged residues } \\
\text { (Arg + Lys) }\end{array}$ & 74 & 76 & 77 & 69 & 69 & 86 & 96 & 72 & 74 & 70 & 75 & 68 & 58 & 66 & 82 & 65 \\
\hline Aliphatic index & 87.06 & 89.17 & 85.75 & 86.66 & 88.60 & 89.73 & 89.42 & 94.70 & 86.93 & 85.82 & 86.92 & 93.95 & 90.47 & 89.62 & 95.53 & 90.75 \\
\hline $\begin{array}{l}\text { Grand average of } \\
\text { hydropathicity } \\
\text { (GRAVY): }\end{array}$ & -0.278 & -0.197 & -0.337 & -0.223 & -0.268 & -0.309 & -0.337 & -0.166 & -0.281 & -0.238 & -0.285 & -0.126 & -0.173 & -0.192 & -0.210 & -0.229 \\
\hline Active site residues & $\begin{array}{c}\mathrm{H}_{368} \\
\mathrm{H}_{373} \\
\mathrm{H}_{551} \\
\mathrm{~N}_{555} \\
\mathrm{I}_{674}\end{array}$ & $\begin{array}{c}\mathrm{H}_{398} \\
\mathrm{H}_{403} \\
\mathrm{H}_{578} \\
\mathrm{~N}_{582} \\
\mathrm{I}_{701}\end{array}$ & $\begin{array}{l}\mathrm{H}_{367} \\
\mathrm{H}_{372} \\
\mathrm{H}_{550} \\
\mathrm{~N}_{554} \\
\mathrm{I}_{673}\end{array}$ & $\begin{array}{c}\mathrm{H}_{398} \\
\mathrm{H}_{403} \\
\mathrm{H}_{578} \\
\mathrm{~N}_{582} \\
\mathrm{I}_{701}\end{array}$ & $\begin{array}{l}\mathrm{H}_{360} \\
\mathrm{H}_{365} \\
\mathrm{H}_{540} \\
\mathrm{~N}_{544} \\
\mathrm{I}_{663}\end{array}$ & $\begin{array}{c}\mathrm{H}_{499} \\
\mathrm{H}_{504} \\
\mathrm{H}_{690} \\
\mathrm{~N}_{694} \\
\mathrm{I}_{839}\end{array}$ & $\begin{array}{l}\mathrm{H}_{518} \\
\mathrm{H}_{523} \\
\mathrm{H}_{709} \\
\mathrm{~N}_{713} \\
\mathrm{I}_{857}\end{array}$ & $\begin{array}{l}\mathrm{H}_{360} \\
\mathrm{H}_{365} \\
\mathrm{H}_{540} \\
\mathrm{I}_{662}\end{array}$ & $\begin{array}{l}\mathrm{H}_{368} \\
\mathrm{H}_{373} \\
\mathrm{H}_{551} \\
\mathrm{~N}_{555} \\
\mathrm{I}_{674}\end{array}$ & $\begin{array}{c}\mathrm{H}_{398} \\
\mathrm{H}_{403} \\
\mathrm{H}_{578} \\
\mathrm{~N}_{582} \\
\mathrm{I}_{701}\end{array}$ & $\begin{array}{c}\mathrm{H}_{367} \\
\mathrm{H}_{372} \\
\mathrm{H}_{550} \\
\mathrm{~N}_{554} \\
\mathrm{I}_{673}\end{array}$ & $\begin{array}{c}\mathrm{H}_{361} \\
\mathrm{H}_{366} \\
\mathrm{H}_{541} \\
\mathrm{H}_{545} \\
\mathrm{I}_{663}\end{array}$ & $\begin{array}{l}\mathrm{H}_{373} \\
\mathrm{H}_{378} \\
\mathrm{H}_{553} \\
\mathrm{I}_{676}\end{array}$ & $\begin{array}{l}\mathrm{H}_{374} \\
\mathrm{H}_{379} \\
\mathrm{H}_{554} \\
\mathrm{I}_{677}\end{array}$ & $\begin{array}{l}\mathrm{H}_{360} \\
\mathrm{H}_{365} \\
\mathrm{H}_{540} \\
\mathrm{I}_{662}\end{array}$ & $\begin{array}{c}\mathrm{H}_{360} \\
\mathrm{H}_{365} \\
\mathrm{H}_{540} \\
\mathrm{~N}_{544} \\
\mathrm{I}_{663}\end{array}$ \\
\hline
\end{tabular}

(TABLE CONTINUED) 


\begin{tabular}{|c|c|c|c|c|c|c|c|c|c|c|}
\hline Proteins & 17 & 18 & 19 & 20 & 21 & 22 & 23 & 24 & 25 & 26 \\
\hline $\begin{array}{c}\text { Number of amino } \\
\text { acids }\end{array}$ & 711 & 864 & 865 & 663 & 663 & 663 & 853 & 677 & 663 & 711 \\
\hline $\begin{array}{l}\text { Molecular Weight } \\
\text { (Da) } \times 10^{3}\end{array}$ & 80.5 & 96.8 & 97.1 & 75.4 & 75.4 & 75.0 & 96.5 & 76.1 & 75.1 & 80.5 \\
\hline Theoretical pl & 6.53 & 5.78 & 6.27 & 5.76 & 6.15 & 5.86 & 5.71 & 5.86 & 6.09 & 6.35 \\
\hline $\begin{array}{c}\text { Number of negatively } \\
\text { charged residues } \\
\text { (Asp + Glu) }\end{array}$ & 69 & 107 & 102 & 79 & 77 & 80 & 115 & 78 & 81 & 72 \\
\hline $\begin{array}{c}\text { Number of positively } \\
\text { charged residues } \\
\text { (Arg + Lys) }\end{array}$ & 65 & 92 & 93 & 67 & 70 & 67 & 99 & 64 & 72 & 66 \\
\hline Aliphatic index & 84.92 & 89.59 & 90.05 & 87.93 & 87.06 & 93.68 & 86.89 & 89.48 & 93.24 & 87.26 \\
\hline $\begin{array}{l}\text { Grand average of } \\
\text { hydropathicity } \\
\text { (GRAVY): }\end{array}$ & -0.188 & -0.280 & -0.293 & -0.211 & -0.188 & -0.153 & -0.361 & -0.170 & -0.190 & -0.148 \\
\hline Active site residues & $\begin{array}{c}\mathrm{H}_{408} \\
\mathrm{H}_{413} \\
\mathrm{H}_{588} \\
\mathrm{~N}_{592} \\
\mathrm{I}_{711}\end{array}$ & $\begin{array}{c}\mathrm{H}_{525} \\
\mathrm{H}_{530} \\
\mathrm{H}_{716} \\
\mathrm{~N}_{720} \\
\mathrm{I}_{864}\end{array}$ & $\begin{array}{c}\mathrm{H}_{527} \\
\mathrm{H}_{532} \\
\mathrm{H}_{718} \\
\mathrm{~N}_{722} \\
\mathrm{I}_{865}\end{array}$ & $\begin{array}{l}\mathrm{H}_{361} \\
\mathrm{H}_{366} \\
\mathrm{H}_{541} \\
\mathrm{I}_{663}\end{array}$ & $\begin{array}{l}\mathrm{H}_{361} \\
\mathrm{H}_{366} \\
\mathrm{H}_{541} \\
\mathrm{I}_{663}\end{array}$ & $\begin{array}{l}\mathrm{H}_{361} \\
\mathrm{H}_{366} \\
\mathrm{H}_{541} \\
\mathrm{I}_{663}\end{array}$ & $\begin{array}{c}\mathrm{H}_{513} \\
\mathrm{H}_{518} \\
\mathrm{H}_{704} \\
\mathrm{~N}_{708} \\
\mathrm{I}_{853}\end{array}$ & $\begin{array}{l}\mathrm{H}_{374} \\
\mathrm{H}_{379} \\
\mathrm{H}_{554} \\
\mathrm{I}_{677}\end{array}$ & $\begin{array}{l}\mathrm{H}_{361} \\
\mathrm{H}_{366} \\
\mathrm{H}_{541} \\
\mathrm{I}_{663}\end{array}$ & $\begin{array}{c}\mathrm{H}_{408} \\
\mathrm{H}_{413} \\
\mathrm{H}_{588} \\
\mathrm{~N}_{592} \\
\mathrm{I}_{711}\end{array}$ \\
\hline
\end{tabular}


Table 4. The amino acid composition of lipoxygenases analyzed.

\begin{tabular}{|c|c|c|c|c|c|c|c|c|c|c|c|c|c|c|c|c|c|c|}
\hline \multirow{2}{*}{$\begin{array}{c}\text { Protein } \\
\text { Amino acids } \\
\text { (aa) }\end{array}$} & \multicolumn{2}{|l|}{1} & \multicolumn{2}{|l|}{2} & \multicolumn{4}{|l|}{3} & \multicolumn{2}{|l|}{5} & \multicolumn{2}{|l|}{6} & \multicolumn{2}{|l|}{7} & \multicolumn{2}{|l|}{8} & \multicolumn{2}{|l|}{9} \\
\hline & $\begin{array}{c}\text { Number } \\
\text { of aa }\end{array}$ & $\%$ & $\begin{array}{c}\text { Number } \\
\text { of aa }\end{array}$ & $\%$ & $\begin{array}{c}\text { Number } \\
\text { of aa }\end{array}$ & $\%$ & $\begin{array}{c}\text { Number } \\
\text { of aa }\end{array}$ & $\%$ & $\begin{array}{c}\text { Number } \\
\text { of aa }\end{array}$ & $\%$ & $\begin{array}{c}\text { Number } \\
\text { of aa }\end{array}$ & $\%$ & $\begin{array}{c}\text { Number } \\
\text { of aa }\end{array}$ & $\%$ & $\begin{array}{c}\text { Number } \\
\text { of aa }\end{array}$ & $\%$ & $\begin{array}{c}\text { Number } \\
\text { of aa }\end{array}$ & $\%$ \\
\hline Ala (A) & 43 & 6.4 & 44 & 6.3 & 40 & 5.9 & 40 & 5.7 & 52 & 7.8 & 56 & 6.7 & 49 & 5.7 & 46 & 6.9 & 43 & 6.4 \\
\hline Arg (R) & 36 & 5.3 & 46 & 6.6 & 34 & 5.1 & 44 & 6.3 & 42 & 6.3 & 37 & 4.4 & 46 & 5.4 & 40 & 6.0 & 32 & 4.7 \\
\hline Asn (N) & 26 & 3.9 & 23 & 3.3 & 26 & 3.9 & 29 & 4.1 & 18 & 2.7 & 40 & 4.8 & 41 & 4.8 & 16 & 2.4 & 25 & 3.7 \\
\hline Asp (D) & 44 & 6.5 & 33 & 4.7 & 43 & 6.4 & 37 & 5.3 & 38 & 5.7 & 46 & 5.5 & 50 & 5.8 & 36 & 5.4 & 42 & 6.2 \\
\hline Cys (C) & 13 & 1.9 & 16 & 2.3 & 13 & 1.9 & 20 & 2.9 & 17 & 2.6 & 4 & 0.5 & 7 & 0.8 & 13 & 2.0 & 12 & 1.8 \\
\hline Gln (Q) & 31 & 4.6 & 22 & 3.1 & 30 & 4.5 & 27 & 3.9 & 40 & 6 & 27 & 3.2 & 25 & 2.9 & 33 & 5.0 & 30 & 4.5 \\
\hline Glu (E) & 48 & 7.1 & 42 & 6 & 48 & 7.1 & 40 & 5.7 & 44 & 6.6 & 55 & 6.6 & 56 & 6.5 & 44 & 6.6 & 47 & 7.0 \\
\hline Gly (G) & 33 & 4.9 & 43 & 6.1 & 33 & 4.9 & 41 & 5.8 & 39 & 5.9 & 53 & 6.3 & 61 & 7.1 & 49 & 7.4 & 33 & 4.9 \\
\hline His (H) & 17 & 2.5 & 22 & 3.1 & 20 & 3 & 21 & 3 & 18 & 2.7 & 25 & 3 & 26 & 3 & 17 & 2.6 & 20 & 3.0 \\
\hline Ile (I) & 44 & 6.5 & 43 & 6.1 & 46 & 6.8 & 43 & 6.1 & 29 & 4.4 & 54 & 6.4 & 50 & 5.8 & 26 & 3.9 & 46 & 6.8 \\
\hline Leu (L) & 59 & 8.8 & 77 & 11 & 59 & 8.8 & 75 & 10.7 & 83 & 12.5 & 86 & 10.3 & 96 & 11.2 & 88 & 13.3 & 59 & 8.8 \\
\hline Lys (K) & 38 & 5.6 & 30 & 4.3 & 43 & 6.4 & 25 & 3.6 & 27 & 4.1 & 49 & 5.8 & 50 & 5.8 & 32 & 4.8 & 42 & 6.2 \\
\hline Met (M) & 16 & 2.4 & 14 & 2 & 14 & 2.1 & 17 & 2.4 & 18 & 2.7 & 15 & 1.8 & 12 & 1.4 & 16 & 2.4 & 15 & 2.2 \\
\hline Phe (F) & 35 & 5.2 & 33 & 4.7 & 34 & 5.1 & 33 & 4.7 & 26 & 3.9 & 30 & 3.6 & 37 & 4.3 & 26 & 3.9 & 35 & 5.2 \\
\hline Pro (P) & 34 & 5.0 & 50 & 7.1 & 32 & 4.8 & 49 & 7 & 43 & 6.5 & 51 & 6.1 & 51 & 6 & 41 & 6.2 & 33 & 4.9 \\
\hline Ser (S) & 33 & 4.9 & 34 & 4.9 & 34 & 5.1 & 34 & 4.9 & 29 & 4.4 & 62 & 7.4 & 56 & 6.5 & 32 & 4.8 & 36 & 5.3 \\
\hline Thr (T) & 30 & 4.5 & 41 & 5.8 & 33 & 4.9 & 40 & 5.7 & 30 & 4.5 & 46 & 5.5 & 47 & 5.5 & 26 & 3.9 & 31 & 4.6 \\
\hline $\operatorname{Trp}(W)$ & 15 & 2.2 & 13 & 1.9 & 14 & 2.1 & 13 & 1.9 & 19 & 2.9 & 14 & 1.7 & 15 & 1.8 & 17 & 2.6 & 15 & 2.2 \\
\hline Tyr (Y) & 30 & 4.5 & 36 & 5.1 & 33 & 4.9 & 36 & 5.1 & 17 & 2.6 & 37 & 4.4 & 31 & 3.6 & 17 & 2.6 & 32 & 4.7 \\
\hline Val (V) & 49 & 7.3 & 39 & 5.6 & 44 & 6.5 & 37 & 5.3 & 34 & 5.1 & 52 & 6.2 & 51 & 6 & 47 & 7.1 & 46 & 6.8 \\
\hline Pyl (0) & 0 & 0 & 0 & 0 & 0 & 0 & 0 & 0 & 0 & 0 & 0 & 0 & 0 & 0 & 0 & 0 & 0 & 0 \\
\hline Sec (U) & 0 & 0 & 0 & 0 & 0 & 0 & 0 & 0 & 0 & 0 & 0 & 0 & 0 & 0 & 0 & 0 & 0 & 0 \\
\hline
\end{tabular}

(TABLE CONTINUED) 


\begin{tabular}{|c|c|c|c|c|c|c|c|c|c|c|c|c|c|c|c|c|c|c|}
\hline \multirow{2}{*}{$\begin{array}{c}\text { Protein } \\
\text { Amino acids } \\
\text { (aa) }\end{array}$} & \multicolumn{2}{|l|}{10} & \multicolumn{2}{|l|}{11} & \multicolumn{2}{|l|}{12} & \multicolumn{2}{|l|}{13} & \multicolumn{2}{|l|}{14} & \multicolumn{2}{|l|}{15} & \multicolumn{2}{|l|}{16} & \multicolumn{2}{|l|}{17} & \multicolumn{2}{|l|}{18} \\
\hline & $\begin{array}{c}\text { Number } \\
\text { of aa }\end{array}$ & $\%$ & $\begin{array}{c}\text { Number } \\
\text { of aa }\end{array}$ & $\%$ & $\begin{array}{c}\text { Number } \\
\text { of aa }\end{array}$ & $\%$ & $\begin{array}{c}\text { Number } \\
\text { of aa }\end{array}$ & $\%$ & $\begin{array}{c}\text { Number } \\
\text { of aa }\end{array}$ & $\%$ & $\begin{array}{c}\text { Number } \\
\text { of aa }\end{array}$ & $\%$ & $\begin{array}{c}\text { Number } \\
\text { of aa }\end{array}$ & $\%$ & $\begin{array}{c}\text { Number } \\
\text { of aa }\end{array}$ & $\%$ & $\begin{array}{c}\text { Number } \\
\text { of aa }\end{array}$ & $\%$ \\
\hline Ala (A) & 39 & 5.6 & 46 & 6.8 & 41 & 6.2 & 49 & 7.2 & 49 & 7.2 & 34 & 5.1 & 46 & 6.9 & 46 & 6.5 & 50 & 5.8 \\
\hline Arg (R) & 43 & 6.1 & 34 & 5.1 & 36 & 5.4 & 35 & 5.2 & 37 & 5.5 & 46 & 6.9 & 39 & 5.9 & 40 & 5.6 & 41 & 4.7 \\
\hline Asn (N) & 32 & 4.6 & 26 & 3.9 & 19 & 2.9 & 22 & 3.3 & 18 & 2.7 & 15 & 2.3 & 19 & 2.9 & 26 & 3.7 & 35 & 4.1 \\
\hline Asp (D) & 35 & 5.0 & 42 & 6.2 & 31 & 4.7 & 28 & 4.1 & 33 & 4.9 & 37 & 5.6 & 37 & 5.6 & 34 & 4.8 & 53 & 6.1 \\
\hline Cys (C) & 20 & 2.9 & 13 & 1.9 & 15 & 2.3 & 11 & 1.6 & 10 & 1.5 & 12 & 1.8 & 14 & 2.1 & 24 & 3.4 & 2 & 0.2 \\
\hline Gln (Q) & 26 & 3.7 & 31 & 4.6 & 37 & 5.6 & 36 & 5.3 & 27 & 4.0 & 34 & 5.1 & 41 & 6.2 & 34 & 4.8 & 24 & 2.8 \\
\hline Glu (E) & 41 & 5.8 & 47 & 7.0 & 44 & 6.6 & 45 & 6.7 & 42 & 6.2 & 41 & 6.2 & 42 & 6.3 & 35 & 4.9 & 54 & 6.2 \\
\hline Gly (G) & 39 & 5.6 & 32 & 4.8 & 45 & 6.8 & 46 & 6.8 & 43 & 6.4 & 41 & 6.2 & 41 & 6.2 & 40 & 5.6 & 64 & 7.4 \\
\hline His (H) & 22 & 3.1 & 19 & 2.8 & 15 & 2.3 & 19 & 2.8 & 24 & 3.5 & 14 & 2.1 & 19 & 2.9 & 18 & 2.5 & 19 & 2.2 \\
\hline lle (I) & 41 & 5.8 & 46 & 6.8 & 29 & 4.4 & 32 & 4.7 & 34 & 5.0 & 26 & 3.9 & 25 & 3.8 & 32 & 4.5 & 64 & 7.4 \\
\hline Leu (L) & 75 & 10.7 & 58 & 8.6 & 86 & 13.0 & 84 & 12.4 & 80 & 11.8 & 91 & 13.7 & 87 & 13.1 & 85 & 12.0 & 83 & 9.6 \\
\hline Lys (K) & 27 & 3.9 & 41 & 6.1 & 32 & 4.8 & 23 & 3.4 & 29 & 4.3 & 36 & 5.4 & 26 & 3.9 & 25 & 3.5 & 51 & 5.9 \\
\hline Met (M) & 18 & 2.6 & 15 & 2.2 & 17 & 2.6 & 11 & 1.6 & 14 & 2.1 & 17 & 2.6 & 18 & 2.7 & 16 & 2.3 & 10 & 1.2 \\
\hline Phe (F) & 34 & 4.9 & 34 & 5.1 & 30 & 4.5 & 32 & 4.7 & 30 & 4.4 & 27 & 4.1 & 27 & 4.1 & 30 & 4.2 & 39 & 4.5 \\
\hline Pro (P) & 50 & 7.1 & 31 & 4.6 & 38 & 5.7 & 53 & 7.8 & 48 & 7.1 & 42 & 6.3 & 44 & 6.6 & 48 & 6.8 & 56 & 6.5 \\
\hline Ser (S) & 33 & 4.7 & 35 & 5.2 & 39 & 5.9 & 41 & 6.1 & 51 & 7.5 & 39 & 5.9 & 29 & 4.4 & 47 & 6.6 & 59 & 6.8 \\
\hline Thr (T) & 40 & 5.7 & 30 & 4.5 & 27 & 4.1 & 37 & 5.5 & 33 & 4.9 & 31 & 4.7 & 35 & 5.3 & 51 & 7.2 & 59 & 6.8 \\
\hline $\operatorname{Trp}(\mathrm{W})$ & 13 & 1.9 & 15 & 2.2 & 18 & 2.7 & 16 & 2.4 & 17 & 2.5 & 13 & 2.0 & 17 & 2.6 & 15 & 2.1 & 14 & 1.6 \\
\hline Tyr (Y) & 35 & 5.0 & 32 & 4.8 & 18 & 2.7 & 18 & 2.7 & 19 & 2.8 & 17 & 2.6 & 16 & 2.4 & 30 & 4.2 & 35 & 4.1 \\
\hline Val (V) & 38 & 5.4 & 46 & 6.8 & 46 & 6.9 & 38 & 5.6 & 39 & 5.8 & 49 & 7.4 & 41 & 6.2 & 35 & 4.9 & 52 & 6.0 \\
\hline Pyl (O) & 0 & 0 & 0 & 0 & 0 & 0 & 0 & 0 & 0 & 0 & 0 & 0 & 0 & 0 & 0 & 0 & 0 & 0 \\
\hline Sec (U) & 0 & 0 & 0 & 0 & 0 & 0 & 0 & 0 & 0 & 0 & 0 & 0 & 0 & 0 & 0 & 0 & 0 & 0 \\
\hline
\end{tabular}

(TABLE CONTINUED) 


\begin{tabular}{|c|c|c|c|c|c|c|c|c|c|c|c|c|c|c|c|c|}
\hline \multirow{2}{*}{$\begin{array}{c}\text { Protein } \\
\text { Amino acids } \\
\text { (aa) }\end{array}$} & \multicolumn{2}{|c|}{19} & \multicolumn{2}{|l|}{20} & \multicolumn{2}{|c|}{21} & \multicolumn{2}{|c|}{22} & \multicolumn{2}{|l|}{23} & \multicolumn{2}{|l|}{24} & \multicolumn{2}{|c|}{25} & \multicolumn{2}{|c|}{26} \\
\hline & $\begin{array}{c}\text { Number } \\
\text { of aa }\end{array}$ & $\%$ & $\begin{array}{c}\text { Number } \\
\text { of aa }\end{array}$ & $\%$ & $\begin{array}{c}\text { Number } \\
\text { of aa }\end{array}$ & $\%$ & $\begin{array}{c}\text { Number } \\
\text { of aa }\end{array}$ & $\%$ & $\begin{array}{c}\text { Number } \\
\text { of aa }\end{array}$ & $\%$ & $\begin{array}{c}\text { Number } \\
\text { of aa }\end{array}$ & $\%$ & $\begin{array}{c}\text { Number } \\
\text { of aa }\end{array}$ & $\%$ & $\begin{array}{c}\text { Number } \\
\text { of aa }\end{array}$ & $\%$ \\
\hline Ala (A) & 55 & 6.4 & 42 & 6.3 & 44 & 6.6 & 44 & 6.6 & 56 & 6.6 & 52 & 7.7 & 42 & 6.3 & 48 & 6.8 \\
\hline Arg (R) & 41 & 4.7 & 34 & 5.1 & 37 & 5.6 & 40 & 6.0 & 33 & 3.9 & 34 & 5.0 & 43 & 6.5 & 38 & 5.3 \\
\hline Asn (N) & 41 & 4.7 & 24 & 3.6 & 23 & 3.5 & 18 & 2.7 & 37 & 4.3 & 17 & 2.5 & 17 & 2.6 & 25 & 3.5 \\
\hline Asp (D) & 48 & 5.5 & 37 & 5.6 & 36 & 5.4 & 35 & 5.3 & 54 & 6.3 & 36 & 5.3 & 38 & 5.7 & 36 & 5.1 \\
\hline Cys (C) & 5 & 0.6 & 16 & 2.4 & 18 & 2.7 & 14 & 2.1 & 3 & 0.4 & 10 & 1.5 & 14 & 2.1 & 27 & 3.8 \\
\hline GIn (Q) & 28 & 3.2 & 34 & 5.1 & 31 & 4.7 & 31 & 4.7 & 24 & 2.8 & 29 & 4.3 & 30 & 4.5 & 32 & 4.5 \\
\hline Glu (E) & 54 & 6.2 & 42 & 6.3 & 41 & 6.2 & 45 & 6.8 & 61 & 7.2 & 42 & 6.2 & 43 & 6.5 & 36 & 5.1 \\
\hline Gly (G) & 61 & 7.1 & 40 & 6.0 & 40 & 6.0 & 46 & 6.9 & 61 & 7.2 & 41 & 6.1 & 48 & 7.2 & 38 & 5.3 \\
\hline His (H) & 24 & 2.8 & 15 & 2.3 & 15 & 2.3 & 19 & 2.9 & 18 & 2.1 & 21 & 3.1 & 18 & 2.7 & 19 & 2.7 \\
\hline Ile (I) & 53 & 6.1 & 25 & 3.8 & 26 & 3.9 & 23 & 3.5 & 62 & 7.3 & 33 & 4.9 & 28 & 4.2 & 34 & 4.8 \\
\hline Leu (L) & 88 & 10.2 & 81 & 12.2 & 78 & 11.8 & 93 & 14.0 & 78 & 9.1 & 80 & 11.8 & 90 & 13.6 & 86 & 12.1 \\
\hline Lys (K) & 52 & 6.0 & 33 & 5.0 & 33 & 5.0 & 27 & 4.1 & 66 & 7.7 & 30 & 4.4 & 29 & 4.4 & 28 & 3.9 \\
\hline Met (M) & 13 & 1.5 & 17 & 2.6 & 21 & 3.2 & 15 & 2.3 & 12 & 1.4 & 14 & 2.1 & 14 & 2.1 & 13 & 1.8 \\
\hline Phe (F) & 34 & 3.9 & 32 & 4.8 & 31 & 4.7 & 30 & 4.5 & 38 & 4.5 & 33 & 4.9 & 29 & 4.4 & 32 & 4.5 \\
\hline Pro (P) & 51 & 5.9 & 39 & 5.9 & 39 & 5.9 & 38 & 5.7 & 49 & 5.7 & 51 & 7.5 & 38 & 5.7 & 49 & 6.9 \\
\hline Ser (S) & 59 & 6.8 & 38 & 5.7 & 40 & 6.0 & 43 & 6.5 & 49 & 5.7 & 49 & 7.2 & 40 & 6.0 & 46 & 6.5 \\
\hline Thr (T) & 47 & 5.4 & 33 & 5.0 & 30 & 4.5 & 26 & 3.9 & 46 & 5.4 & 31 & 4.6 & 29 & 4.4 & 45 & 6.3 \\
\hline Trp (W) & 14 & 1.6 & 16 & 2.4 & 16 & 2.4 & 17 & 2.6 & 16 & 1.9 & 17 & 2.5 & 18 & 2.7 & 14 & 2.0 \\
\hline Tyr (Y) & 37 & 4.3 & 21 & 3.2 & 20 & 3.0 & 16 & 2.4 & 42 & 4.9 & 18 & 2.7 & 15 & 2.3 & 29 & 4.1 \\
\hline Val (V) & 60 & 6.9 & 44 & 6.6 & 44 & 6.6 & 43 & 6.5 & 48 & 5.6 & 39 & 5.8 & 40 & 6.0 & 36 & 5.1 \\
\hline Pyl (0) & 0 & 0 & 0 & 0 & 0 & 0 & 0 & 0 & 0 & 0 & 0 & 0 & 0 & 0 & 0 & 0 \\
\hline Sec (U) & 0 & 0 & 0 & 0 & 0 & 0 & 0 & 0 & 0 & 0 & 0 & 0 & 0 & 0 & 0 & 0 \\
\hline
\end{tabular}


Cengiz Şahin S, Cavas L. JOTCSA. 2021; 8(1): 79-102.

In order to obtain genetic relationships among LOXs studied, a phylogenetic tree is constructed in Figure 1. According to phylogenetic tree, soybean LOXs are within separate clade compared to mammalian LOXs. The human LOXs are in the same clade with the mouse, rat and golden hamster. The sequence identity between the plant and mammalian LOXs are reported as 21\%-27\% only while it is $43 \%-86 \%$ for plant LOXs and $39 \%-$ $93 \%$ for mammalian ones (47). The phylogenetic tree shows that soybean LOXs are different compared to mammalian LOXs.
The similarities among sequences of LOXs studied are analyzed by Clustal Omega for multiple alignments. According to these results, the best similarities are observed with the score of 98 between 5-LO (rat) - 5-LO (mouse). Besides, 5-LO (rat), 5-LO (mouse) and 5-LO (golden hamster) can be a better model for 5-LO (human) as the scores of Clustal Omega are observed 93. 12R-LOX (human) can be best represented by 12R-LOX (mouse) and 12R-LOX (rat) with the score of 86. 12S-LOX (human) can be best represented by 125 LOX (mouse) with the score of 86. 15-LOX (human) can be best represented by 15-LOX (pig or bovine) with the score of 87 (Table 5).

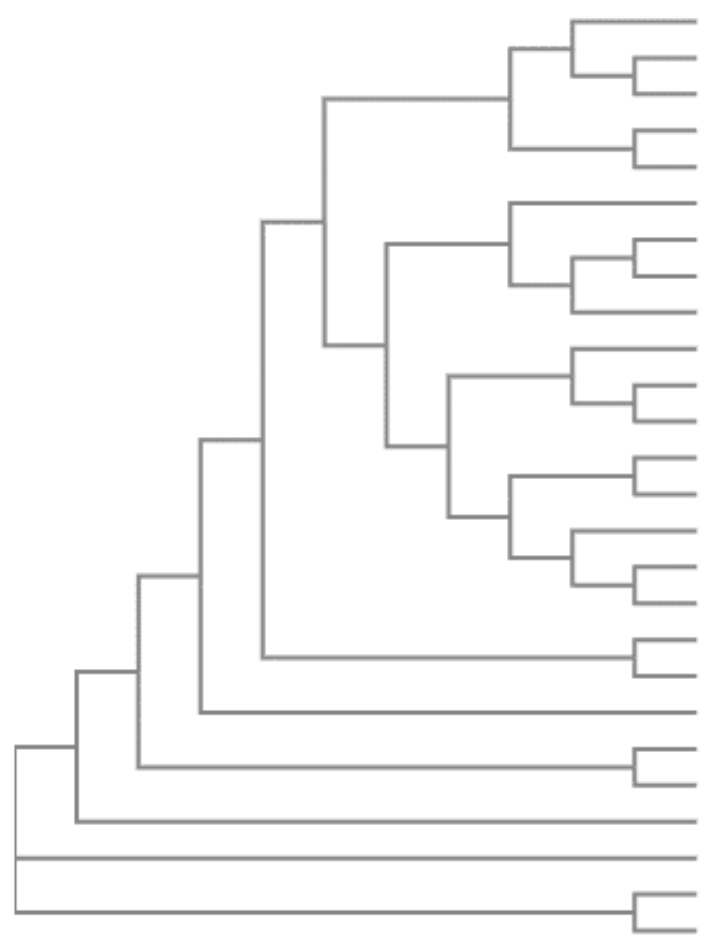

LOXX_SOYBN 0.13987

LOX1_SOYBN 0.08338

LOX2 SOYBN 0.07111

LOX4_SOYBN 0.13451

LOX3_SOYBN 0.12196

LOX5_HUMAN 0.02801

LOX5_RAT 0.01209

LOX5_MOUSE 0.0102

LOX5_MESAU 0.01894

LX15B_HUMAN 0.09954

ALOX8_MOUSE 0.05044

LX15B_RAT 0.05739

LOXE3_HUMAN 0.06007

LOXE3_MOUSE 0.05526

LX12B_HUMAN 0.06577

LX12B_MOUSE 0.01376

LX12B_RAT 0.01477

LOX12_HUMAN 0.06992

LOX12_MOUSE 0.07488

LX12E MOUSE 0.18582

LOX15_MOUSE 0.03107

LOX15_RAT 0.02775

LOX15_RABIT 0.11069

LOX15_HUMAN 0.05808

LOX15 PIG 0.05427

LOX15_BOVIN 0.05131

Figure 1. Phylogenetic tree of lipoxygenases examined. 
Table 5. The similarity scores of lipoxygenases obtained from Clustal Omega analysis.

\begin{tabular}{|c|c|c|c|c|c|c|c|c|c|c|c|c|c|c|}
\hline & & 1 & 2 & 3 & 4 & 5 & 6 & 7 & 8 & 9 & 10 & 11 & 12 & 13 \\
\hline 1 & LOXX_SOYBN & 100 & 67.84 & 71.09 & 70.3 & 71.93 & 26.4 & 26.4 & 26.79 & 25.51 & 25.04 & 24.42 & 25.86 & 26.44 \\
\hline 2 & LOX4_SOYBN & 67.84 & 100 & 74.35 & 66.51 & 67.45 & 24.73 & 24.73 & 26.68 & 23.83 & 24.14 & 23.52 & 24.49 & 25.39 \\
\hline 3 & LOX3_SOYBN & 71.09 & 74.35 & 100 & 72.25 & 75.03 & 26.36 & 27.75 & 27.99 & 25.93 & 25.78 & 25.78 & 26.28 & 27.17 \\
\hline 4 & LOX1_SOYBN & 70.3 & 66.51 & 72.25 & 100 & 84.55 & 24.53 & 24.69 & 27.12 & 25.2 & 24.57 & 24.26 & 25.24 & 25.98 \\
\hline 5 & LOX2_SOYBN & 71.93 & 67.45 & 75.03 & 84.55 & 100 & 26.13 & 25.82 & 27.77 & 26.17 & 25.23 & 24.77 & 25.43 & 26.64 \\
\hline 6 & LOX12_HUMAN & 26.4 & 24.73 & 26.36 & 24.53 & 26.13 & 100 & 85.52 & 59.76 & 57.4 & 58.61 & 61.78 & 65.51 & 65.71 \\
\hline 7 & LOX12_MOUSE & 26.4 & 24.73 & 27.75 & 24.69 & 25.82 & 85.52 & 100 & 60.06 & 58.16 & 58.16 & 60.57 & 63.69 & 64.35 \\
\hline 8 & LX12E_MOUSE & 26.79 & 26.68 & 27.99 & 27.12 & 27.77 & 59.76 & 60.06 & 100 & 59.52 & 59.67 & 62.39 & 66.41 & 65.86 \\
\hline 9 & LOX15_MOUSE & 25.51 & 23.83 & 25.93 & 25.2 & 26.17 & 57.4 & 58.16 & 59.52 & 100 & 94.12 & 71.49 & 73.72 & 70.74 \\
\hline 10 & LOX15_RAT & 25.04 & 24.14 & 25.78 & 24.57 & 25.23 & 58.61 & 58.16 & 59.67 & 94.12 & 100 & 71.79 & 74.77 & 71.79 \\
\hline 11 & LOX15_RABIT & 24.42 & 23.52 & 25.78 & 24.26 & 24.77 & 61.78 & 60.57 & 62.39 & 71.49 & 71.79 & 100 & 81.27 & 79.34 \\
\hline 12 & LOX15_HUMAN & 25.86 & 24.49 & 26.28 & 25.24 & 25.43 & 65.51 & 63.69 & 66.41 & 73.72 & 74.77 & 81.27 & 100 & 86.56 \\
\hline 13 & LOX15_PIG & 26.44 & 25.39 & 27.17 & 25.98 & 26.64 & 65.71 & 64.35 & 65.86 & 70.74 & 71.79 & 79.34 & 86.56 & 100 \\
\hline 14 & LOX15_BOVIN & 26.28 & 24.92 & 27.33 & 25.51 & 26.17 & 65.11 & 64.35 & 66.31 & 72.25 & 73 & 78.73 & 86.86 & 89.44 \\
\hline 15 & LOX5_HUMAN & 28.91 & 28.96 & 29.08 & 25.97 & 27.99 & 42.86 & 42.69 & 40.43 & 41.69 & 41.69 & 40.03 & 41.2 & 42.19 \\
\hline 16 & LOX5_RAT & 27.37 & 28.42 & 27.22 & 24.54 & 26.53 & 40.85 & 41.01 & 38.93 & 40.55 & 40.24 & 38.26 & 40.09 & 40.85 \\
\hline 17 & LOX5_MOUSE & 27.79 & 28.53 & 27.63 & 24.96 & 26.95 & 40.94 & 41.1 & 39.02 & 40.79 & 40.49 & 38.36 & 40.18 & 40.79 \\
\hline 18 & LOX5_MESAU & 27.68 & 28.57 & 27.52 & 24.69 & 26.84 & 41.31 & 41.16 & 38.47 & 40.24 & 40.09 & 38.11 & 39.48 & 40.7 \\
\hline 19 & LX15B_HUMAN & 27.37 & 26.19 & 27.33 & 25.42 & 26.07 & 38.05 & 38.66 & 36.28 & 38.36 & 38.2 & 37.75 & 38.36 & 38.66 \\
\hline 20 & ALOX8_MOUSE & 27.63 & 26.91 & 27.59 & 25.38 & 26.19 & 38.05 & 38.81 & 36.89 & 38.36 & 38.66 & 36.99 & 38.05 & 38.2 \\
\hline 21 & LX15B_RAT & 26.56 & 25.54 & 26.37 & 24.77 & 25.88 & 37.6 & 37.9 & 35.82 & 37.44 & 37.29 & 36.07 & 36.99 & 37.14 \\
\hline 22 & LOXE3_HUMAN & 27.22 & 26.71 & 27.33 & 25.34 & 26.41 & 37.78 & 37.48 & 35.71 & 37.63 & 37.63 & 37.78 & 37.48 & 37.78 \\
\hline 23 & LOXE3_MOUSE & 27.96 & 27 & 27.47 & 26.23 & 27.45 & 38.09 & 37.48 & 37.08 & 38.54 & 38.09 & 37.48 & 37.78 & 37.63 \\
\hline 24 & LX12B_HUMAN & 25.98 & 26.05 & 25.34 & 23.6 & 25 & 38.09 & 37.18 & 34.65 & 35.96 & 36.12 & 35.05 & 35.51 & 34.6 \\
\hline 25 & LX12B_MOUSE & 25.08 & 25 & 24.89 & 23.3 & 24.7 & 37.33 & 36.57 & 34.5 & 35.36 & 35.36 & 35.36 & 35.05 & 34.6 \\
\hline 26 & LX12B_RAT & 25.08 & 25.15 & 24.74 & 23.3 & 24.55 & 37.03 & 36.42 & 34.5 & 35.2 & 35.36 & 35.05 & 34.75 & 33.99 \\
\hline
\end{tabular}

(TABLE CONTINUE) 


\begin{tabular}{|c|c|c|c|c|c|c|c|c|c|c|c|c|c|c|}
\hline & & 14 & 15 & 16 & 17 & 18 & 19 & 20 & 21 & 22 & 23 & 24 & 25 & 26 \\
\hline 1 & LOXX_SOYBN & 26.28 & 28.91 & 27.37 & 27.79 & 27.68 & 27.37 & 27.63 & 26.56 & 27.22 & 27.96 & 25.98 & 25.08 & 25.08 \\
\hline 2 & LOX4_SOYBN & 24.92 & 28.96 & 28.42 & 28.53 & 28.57 & 26.19 & 26.91 & 25.54 & 26.71 & 27 & 26.05 & 25 & 25.15 \\
\hline 3 & LOX3_SOYBN & 27.33 & 29.08 & 27.22 & 27.63 & 27.52 & 27.33 & 27.59 & 26.37 & 27.33 & 27.47 & 25.34 & 24.89 & 24.74 \\
\hline 4 & LOX1_SOYBN & 25.51 & 25.97 & 24.54 & 24.96 & 24.69 & 25.42 & 25.38 & 24.77 & 25.34 & 26.23 & 23.6 & 23.3 & 23.3 \\
\hline 5 & LOX2_SOYBN & 26.17 & 27.99 & 26.53 & 26.95 & 26.84 & 26.07 & 26.19 & 25.88 & 26.41 & 27.45 & 25 & 24.7 & 24.55 \\
\hline 6 & LOX12_HUMAN & 65.11 & 42.86 & 40.85 & 40.94 & 41.31 & 38.05 & 38.05 & 37.6 & 37.78 & 38.09 & 38.09 & 37.33 & 37.03 \\
\hline 7 & LOX12_MOUSE & 64.35 & 42.69 & 41.01 & 41.1 & 41.16 & 38.66 & 38.81 & 37.9 & 37.48 & 37.48 & 37.18 & 36.57 & 36.42 \\
\hline 8 & LX12E_MOUSE & 66.31 & 40.43 & 38.93 & 39.02 & 38.47 & 36.28 & 36.89 & 35.82 & 35.71 & 37.08 & 34.65 & 34.5 & 34.5 \\
\hline 9 & LOX15_MOUSE & 72.25 & 41.69 & 40.55 & 40.79 & 40.24 & 38.36 & 38.36 & 37.44 & 37.63 & 38.54 & 35.96 & 35.36 & 35.2 \\
\hline 10 & LOX15_RAT & 73 & 41.69 & 40.24 & 40.49 & 40.09 & 38.2 & 38.66 & 37.29 & 37.63 & 38.09 & 36.12 & 35.36 & 35.36 \\
\hline 11 & LOX15_RABIT & 78.73 & 40.03 & 38.26 & 38.36 & 38.11 & 37.75 & 36.99 & 36.07 & 37.78 & 37.48 & 35.05 & 35.36 & 35.05 \\
\hline 12 & LOX15_HUMAN & 86.86 & 41.2 & 40.09 & 40.18 & 39.48 & 38.36 & 38.05 & 36.99 & 37.48 & 37.78 & 35.51 & 35.05 & 34.75 \\
\hline 13 & LOX15_PIG & 89.44 & 42.19 & 40.85 & 40.79 & 40.7 & 38.66 & 38.2 & 37.14 & 37.78 & 37.63 & 34.6 & 34.6 & 33.99 \\
\hline 14 & LOX15_BOVIN & 100 & 41.69 & 40.85 & 40.94 & 40.24 & 39.57 & 38.05 & 37.6 & 37.63 & 37.94 & 35.51 & 35.05 & 34.6 \\
\hline 15 & LOX5_HUMAN & 41.69 & 100 & 92.99 & 93.16 & 93.31 & 43.64 & 44.46 & 43.8 & 41.22 & 42.36 & 40.07 & 39.74 & 39.57 \\
\hline 16 & LOX5_RAT & 40.85 & 92.99 & 100 & 97.77 & 96.14 & 42.53 & 43.59 & 42.23 & 40.63 & 41.83 & 40.03 & 40.18 & 40.03 \\
\hline 17 & LOX5_MOUSE & 40.94 & 93.16 & 97.77 & 100 & 96.58 & 42.92 & 43.52 & 42.47 & 41.02 & 42.22 & 40.27 & 40.42 & 40.27 \\
\hline 18 & LOX5_MESAU & 40.24 & 93.31 & 96.14 & 96.58 & 100 & 42.68 & 43.74 & 42.53 & 40.93 & 42.13 & 39.73 & 39.73 & 39.73 \\
\hline 19 & LX15B_HUMAN & 39.57 & 43.64 & 42.53 & 42.92 & 42.68 & 100 & 78.11 & 80.77 & 55.07 & 54.63 & 50.9 & 50.75 & 51.04 \\
\hline 20 & ALOX8_MOUSE & 38.05 & 44.46 & 43.59 & 43.52 & 43.74 & 78.11 & 100 & 89.22 & 53.73 & 53.58 & 50.3 & 49.1 & 49.4 \\
\hline 21 & LX15B_RAT & 37.6 & 43.8 & 42.23 & 42.47 & 42.53 & 80.77 & 89.22 & 100 & 52.09 & 52.39 & 50.6 & 49.7 & 50.15 \\
\hline 22 & LOXE3_HUMAN & 37.63 & 41.22 & 40.63 & 41.02 & 40.93 & 55.07 & 53.73 & 52.09 & 100 & 88.47 & 54.92 & 53.78 & 53.21 \\
\hline 23 & LOXE3_MOUSE & 37.94 & 42.36 & 41.83 & 42.22 & 42.13 & 54.63 & 53.58 & 52.39 & 88.47 & 100 & 55.49 & 54.21 & 53.64 \\
\hline 24 & LX12B_HUMAN & 35.51 & 40.07 & 40.03 & 40.27 & 39.73 & 50.9 & 50.3 & 50.6 & 54.92 & 55.49 & 100 & 86.02 & 86.59 \\
\hline 25 & LX12B_MOUSE & 35.05 & 39.74 & 40.18 & 40.42 & 39.73 & 50.75 & 49.1 & 49.7 & 53.78 & 54.21 & 86.02 & 100 & 97.15 \\
\hline 26 & LX12B_RAT & 34.6 & 39.57 & 40.03 & 40.27 & 39.73 & 51.04 & 49.4 & 50.15 & 53.21 & 53.64 & 86.59 & 97.15 & 100 \\
\hline
\end{tabular}


Cengiz Şahin S, Cavas L. JOTCSA. 2021; 8(1): 79-102.

The sequence alignments may present the deletion and conserved amino acids residues during the evolution process. According to the results from the sequence alignments, a deletion site which consists of 177 amino acids is observed in 15-LOX (human) compared to soybean LOX-1 (Table 6a). On the other hand, the high similarities are seen between 15-LOX (human) and 15-LOX (rabbit). There is only one amino acid deletion which is localized at 90th position of the 15-LOX (human) compared to 15-LOX (rabbit). There are 535 conserved residues, 70 residues with very similar physical characteristics, 21 residues with similar physical characteristics in LOXs analyzed which is presented in Table $6 \mathrm{~b}$. The results presented in Table 7 that there is an important structural similarity between soybean LOX-1 and 15-LOX (human) enzymes and the alpha-helical structure is more common model than $\beta$-strand. It is important to note that the enzymes compared have remarkable similarity, although there is quite a difference between the amino acid residues of these sequences. As can be seen from that figure the same structured regions contained 135 conserved amino acid residues. Moreover, all of the amino acid residues replaced in active site have the same secondary structure prediction (Table 7). Amagata et al (48) investigated the inhibitory activities and the selectivity of some compounds isolated from marine sponge on soybean LOX-1 and 15-LOX (human). The different $I C_{50}$ values obtained for these compounds and the variations in selectivity presented that the structures of studied enzymes were different. Prigge et al (49) use the identified molecular structure of soybean LOX-1 as a model in order to develop the possible structures of human 5-, 12-, and 15- LOXs. Although the structures of human enzymes were not identified fully, the molecular models of active sites that are highly conserved of these enzymes were studied by Prigge et al (49). Besides, the differences among sequences were analyzed in details. There has not been any report which identified the 3D structure of 15-LOX (human). The known 3D structure of LOX from mammalians is only reticulocyte-type 15-LOX (rabbit). According to alignments between the sequences of soybean LOX-1, 15-LOX (human) and 15-LOX (rabbit), the 3D structure of 15-LOX (rabbit) can be a better model for 15-LOX (human) than that of soybean LOX-1. The Clustal Omega score was 81 between 15-LOX (human) and 15-LOX (rabbit). These results are well in line with the results of Gillmor et al (50) and Choi et al (51) who reported that the crystal structure of 15-LOX (rabbit) had an important role for understanding the properties of mammalian LOXs. 
Table 6a. Sequence alignments between Soybean LOX-1 - 15-LOX (human) ((*) conserved, (:) very similar, (.) somewhat similar physicochemical character). SP|P08170|LOX1_SOYBN MFSAGHKIKGTVVLMPKNELEVNPDGSAVDNLNAFLGRSVSLQLISATKADAHGKGKVGK 60 SP |P16050| LOX15_HUMAN --SP|P08170|LOX1_SOYBN DTFLEGINTSLPTLGAGESAFNIH-FEWDGSMGIPGAFYIKNYMQVVFFL̈KSLTLEAISN 119 SP|P16050|LOX15_HUMAN RLWPA--------RGK-ETELKVEVPEYLGPLLFVKLRKRHLLKDDAWFCNWISVQGPGA 88

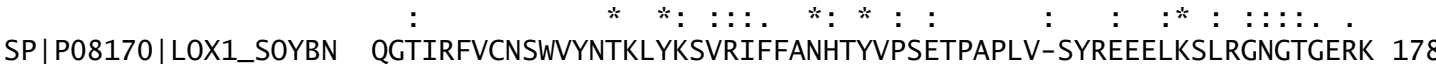
SP|P16050|LOX15_HUMAN GDEVRFPCYRWVEGNGVLSLPE----GTGRTVGEDPQGLFQKHREEE----------LE 133 SP|P08170|LOX1_SOYBN EYDRIYDYDVYNDLGNPDKSEKLARPVLGGSSTFPYPRRGRTGRGPTVTDPNTEKQGEVF 238

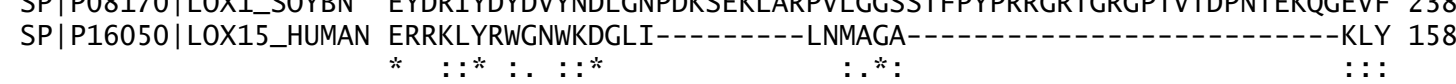

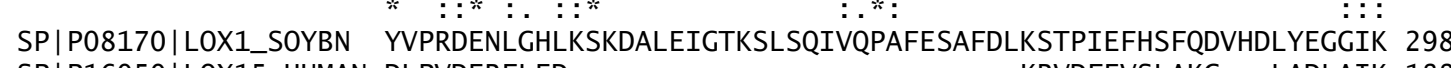
$\begin{aligned} \text { SP|P16050|LOX15_HUMAN DLPVDERFLED-- } & \text { :***: . }\end{aligned}$ $*:: * \quad: \quad *: . * *$

SP|P08170|LOX1_SOYBN LPRDVISTIIPLPVIKELYRTDGQHILKFPQPHVVQVSQSAWMTDEEFAREMIAGVNPCV 358 SP|P16050|LOX15_HUMAN DSLNVLTC----WKDL--DDFNRIFWCGQSKLAERVRDSWKEDALFGYFLNGANPVV 240 SP|P08170|LOX1_SOYBN IRGLEEFPPKSNLDPAIYGDQSSKITADSLDLDGYTMDEALGSRRLFMLDYHDIFMPYVR 418 SP|P16050|LOX15_HUMAN LRRSAHLPARLVFPPGMEELQAQ---------LEKELEGGTLFEADFSLLD-GIKA 286

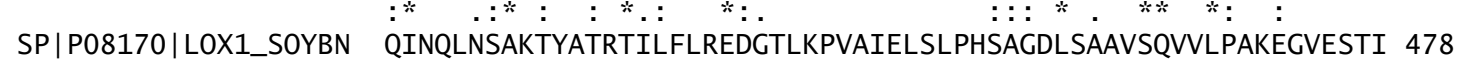
$\begin{array}{lll}\text { SP|P08170|LOX1_SOYBN } & \text { QINQLNSAKTYATRTILFLREDGTLKPVAIELSLPHSAGDLSAAVSQVVLPAKECVESTI } 478 \\ \text { SP|P16050|LOX15_HUMAN NVILCSQQHLAAPLVMLKLQPDGKLLPMVIQLQLPRTCSPPPP----LFLPT---DPPMA } 339\end{array}$

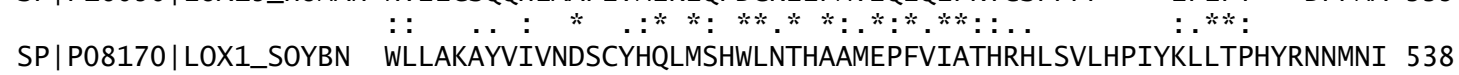
SP|P16050|LOX15_HUMAN WLLAKCWVRSSDFQLHELQSHLLRGHLMAEVIVVATMRCLPSIHPIFKLIIPHLRYTLEI 399 $* * * *:: * * *: * * *: * *: *: * * * *: * * *: * *: * * *::: *$

SP|P08170|LOX1_SOYBN NALARQSLINANGIIETTFLPSKYS-VEMSSAVYKNWVFTDQALPADLIKRGVAIKDPST 597 SP|P16050|LOX15_HUMAN NVRARTGLVSDMGIFDQIMSTGGGGHVQLLKQAGAFLTYSSFCPPDDLADRGL------ 452

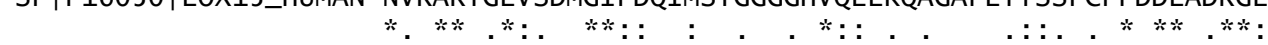

SP|P08170|LOX1_SOYBN PHGVRLLIEDYPYAADGLEIWAAIKTWVQEYVPLYYARDDDVKNDSELQHWWKEAVEKGH 657 SP | P16050| LOX15_HUMAN ----LGVKSSFYAQDALRLWEIIYRYVEGIVSLHYKTDVAVKDDPELQTWCREITEIGL 507

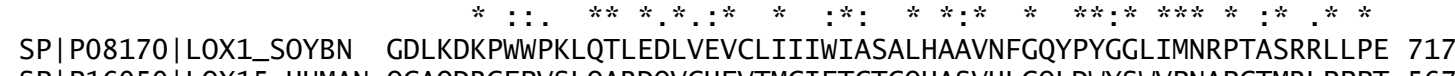
SP|P16050|LOX15_HUMAN QGAQDRGFPVSLQARDQVCHFVTMCIFTCTGQHASVHLGQLDWYSWVPNAPCTMRLPPPT 567 $: *:: * *::: \ldots: *:::^{*} * *: *: * *::: * *: * * *$

SP|P08170|LOX1_SOYBN KGTPEYEEMINNHEKAYLRTITSKLPTLISLS---VIEILSTHASDEVYLGQRDNPHWT 773 SP |P16050 | LOX15_HUMAN TKDATLE--.-1.-TVMATLPNFHQASLQMSITWQLGRRQPVMVAVGQHEEE-YF 614 SP|P08170|LOX1_SOYBN SंDSKALQAFQKFGNKLKEIEEKLVRRNNDPSLQGNRLCPVQLPY' SP|P16050|LOX15_HUMAN SGPEPKAVLKKFREELAALDKEIEIRN--------AKLDMPYEYLRPSVVENSV---- 660 SP|P08170|LOX1_SOYBN PNSISI $839 *:: * \quad:::: * * * \quad:::: * * * * * * . *$ SP|P16050|LOX15_HUMAN ---AI 662 


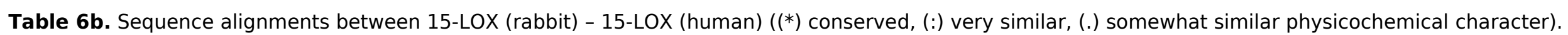

SP|P16050|LOX15_HUMAN MGLYRIRVSTGASLYAGSNNQVQLWLVGQHGEAALGKRLWPARGKETELKVEVPEYLGPL 60 SP|P12530|LOX15_RABIT MGVYRVCVSTGASIYAGSKNKVELWLVGQHGEVELGSCLRPTRNKEEEFKVNVSKYLGSL 60

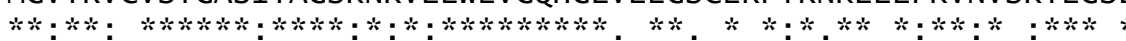

SP|P16050|L0X15_HUMAN LFVKLRKRHLLKDDAWFCNWISVQGPGAG-DEVRFPCYRWVEGNGVLSLPEGTGRTVGED 119 SP|P12530|LOX15_RABIT LFVRLRKKHFLKEDAWFCNWISVQALGAAEDKYWFPCYRWVVGDGVQSLPVGTGCTTVGD 120

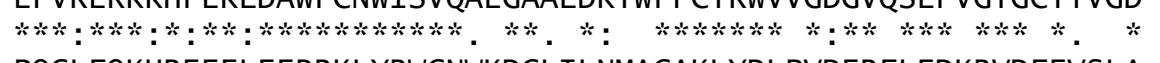

SP|P16050|LOX15_HUMAN PQGLFQKHREEELEERRKLYRWGNWKDGLILNMAGAKLYDLPVDERFLEDKRVDFEVSLA 179 SP|P12530|LOX15_RABIT PQGLFQKHREQELEERRKLYQWGSWKEGLILNVAGSKLTDLPVDERFLEDKKIDFEASLA 180

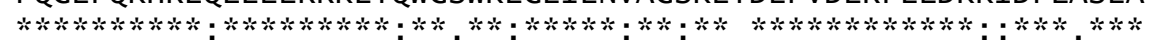

SP|P16050|LOX15_HUMAN KGLADLAIKDSLNVLTCWKDLDDFNRIFWCGQSKLAERVRDSWKEDALFGYQFLNGANPV 239 SP|P12530|LOX15_RABIT WGLAELALKNSLNILAPWKTLDDFNRIFWCGRSKLARRVRDSWQEDSLFGYQFLNGANPM 240 $* * *: * *: *: * *: *: * * * * * * * * * * * * *: * * * * ; * * * * *: * *: * * * * * * * * * * * *$

SP|P16050|LOX15_HUMAN VLRRSAHLPARLVFPPGMEELOAOLEKELEGGTLFEADFSLLDGIKANVILCSOOHLAAP 299 SP|P12530|LOX15_RABIT LLRRSVQLPARLVFPPGMEELQAQLEKELKAGTLFEADFALLDNIKANVILYCQQYLAAP 300

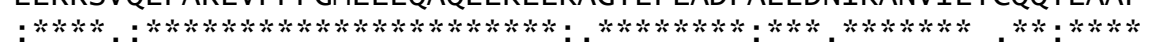

SP|P16050|LOX15_HUMAN LVMLKLOPDGKLLPMVIOLOLPRTCSPPPPLFLPTDPPMAWLLAKCWVRSSDFQLHELQS 359 SP|P12530|LOX15_RABIT LVMLKLQPDGKLMPMVIQLHLPKIGSSPPPLFLPTDPPMVWLLAKCWVRSSDFOVHELNS 360

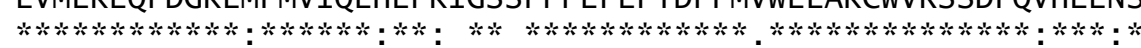

SP|P16050|LOX15_HUMAN HLLRGHLMAEVIVVATMRCLPSIHPIFKLIIPHLRYTLEINVRARTGLVSDMGIFDQIMS 419 SP|P12530|LOX15_RABIT HLLRGHLMAEVFTVATMRCLPSIHPVFKLIVPHLRYTLEINVRARNGLVSDFGIFDQIMS 420

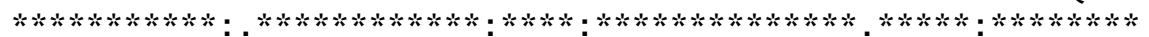

SP|P16050|LOX15_HUMAN TGGGGHVQLLKQAGAFLTYSSFCPPDDLADRGLLGVKSSFYAQDALRLWEIIYRYVEGIV 479 SP|P12530|LOX15_RABIT TGGGGHVQLLQQAGAFLTYRSFCPPDDLADRGLLGVESSFYAQDALRLWEIISRYVQGIM 480 $* * * * * * * * * * ; * * * * * * * * * * * * * * * * * * * * * * *: * * * * * * * * * * * * * * * * * * *: * *:$

SP|P16050|LOX15_HUMAN SLHYKTDVAVKDDPELOTWCREITEIGLOGAODRGFPVSLOARDOVCHFVTMCIFTCTCO 539 SP|P12530|LOX15_RABIT GLYYKTDEAVRDDLELQSWCREITEIGLQGAQKQGFPTSLQSVAQACHFVTMCIFTCTCQ 540

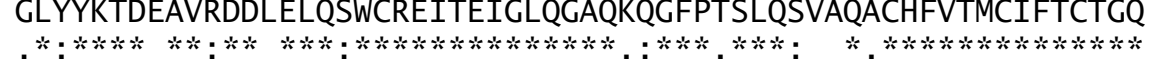

SP|P16050|LOX15_HUMAN HASVHLGQLDWYSWVPNAPCTMRLPPPTTKDATLETVMATLPNFHQASLQMSITWQLGRR 599 SP|P12530|LOX15_RABIT HSSIHLGQLDWFTWVPNAPCTMRLPPPTTKDATLETVMATLPNLHQSSLQMSIVWQLGRD 600

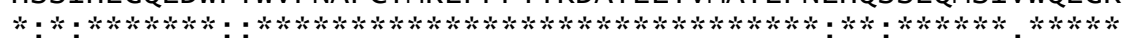

SP|P16050|LOX15_HUMAN QPVMVAVGQHEEEYFSGPEPKAVLKKFREELAALDKEIEIRNAKLDMPYEYLRPSVVENS 659 SP|P12530|LOX15_RABIT QPIMVPLGQHQEEYFSGPEPRAVLEKFREELAIMDKEIEVRNEKLDIPYEYLRPSIVENS 660 $* *: * *: * * *: * * * * * * * * *: * * * ; * * * * * *: ; * * * *: * * * * *: * * * * * * * * ; * * * *$

SP|P16050|LOX15 HUMAN VAI 662

SP|P12530|LOX15_RABIT VAI 663

$* * *$ 


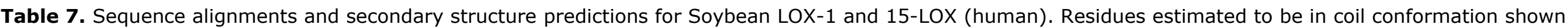
in yellow; residues estimated to be in $\beta$-sheet conformation shown in blue and residues estimated to be in alpha-helical conformation shown in green.

SP|P16050|LOX15_HUMAN - - . - . - . - MGLYRIRVSTGA - . - -SLYAGSNNQVQLW - -LVGQHGEAALGK 37 SP|PO8170|LOX1_SOYBN MFSAGHKIKGTVVLMPKNELEVNPDGSAVDNLNAFLGRSVSLQLISATKADAHGKGKVGK 60 SP|P16050|LOX15_HUMAN RLWPA- - - - - -RGK-ETELKVEVPEYLGPLLFVKLRKRHLLKDDAWFCNWISVQGPGA 88 SP|P๑8170|LOX1_SOYBN DTFLEGINTSLPTLGAGESAFNIH-FEWDGSMGIPGAFYIKNYMQVEFFLKSLTLEAISN 119 ${ }^{*}::::{ }^{*}:{ }^{*}:: \quad: \quad: \quad:^{*}:::::$

SP|P16050|LOX15_HUMAN GDEVRFPCYRWVEGNGVLSLPE - - - GTGRTVGEDPQGLFQKHREEE- - - - - - - - - LE 133 SP|P08170|LOX1_SOYBN QGTIRFVCNSWVYNTKLYKSVRIFFANHTYVPSETPAPLV-SYREEELKSLRGNGTGERK 178

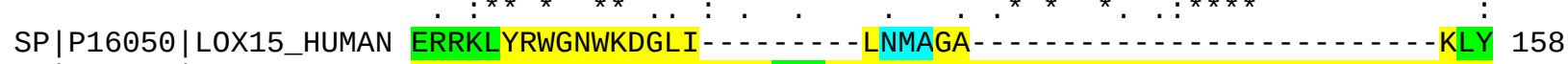
SP|P08170|LOX1_SOYBN EYDRIYDYDVYNDLGNPDKSEKLARPVLGGSSTFPYPRRGRTGRGPTVTDPNTEKQGEVF 238

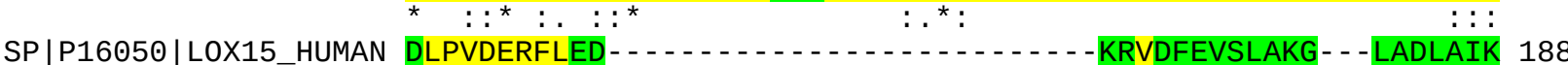
SP|P08170|LOX1_SOYBN YVPRDENLGHLKSKDALEIGTKSLSQIVQPAFESAFDLKSTPIEFHSFQDVHDLYEGGIK 298

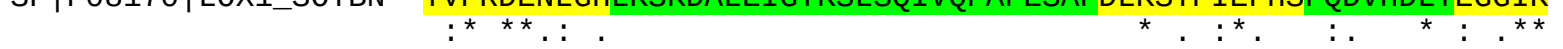
SP|P16050|LOX15_HUMAN DSLNVLTC-- - - -WKDL- -DDFNRIFWCGQSKLAERVRDSWKEDALFGYQFLNGANPVV 240 SP|P08170|LOX1_SOYBN LPRDVISTIIPLPVIKELYRTDGQHILKFPQPHVVQVSQSAWMTDEEFAREMIAGVNPCV 358

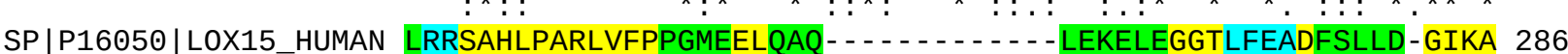
SP|PO8170|LOX1_SOYBN IRGLEEFPPKSNLDPAIYGDQSSKITADSLDLDGYTMDEALGSRRLFMLDYHDIFMPYVR 418 $:^{*} \quad:^{*}: \quad:{ }^{*} .:{ }^{*}: \quad::^{*} \cdot{ }^{*}{ }^{*}:$

SP|P16050|LOX15_HUMAN NVILCSQQHLAAPLVMLKLQPDGKLLPMVIQLQLPRTGSPPPP - - - LFLPT - - -DPPMA 339 SP|P08170|LOX1_SOYBN QINQLNSAKTYATRTILFLREDGTLKPVAIELSLPHSAGDLSAAVSQVVLPAKEGVESTI 478 SP|P16050|LOX15_HUMAN WLLAKCWVRSSDFQLHELQSHLLRGHLMAEVIVVATMRCLPSIHPIFKLIIPHLRYTLEI 399 SP|P08170|LOX1_SOYBN WLLAKAYVIVNDSCYHQLMSHWLNTHAAMEPFVIATHRHLSVLHPIYKLLTPHYRNNMNI 538 SP|P16050|LOX15_HUMAN NVRARTGLVSDMGIFDQIMSTGGGGHVQLLKQAGAFLTYSSFCPPDDLADRGL - _ - _ 452 SP|P08170|LOX1_SOYBN NALARQSLINANGIIETTFLPSKYS-VEMSSAVYKNWVFTDQALPADLIKRGVAIKDPST 597

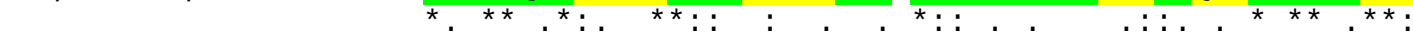

SP|P16050|LOX15_HUMAN - - - -LGVKSSFYAQDALRLWEIIYRYVEGIVSLHYKTDVAVKDDPELQTWCREITEIGL 507

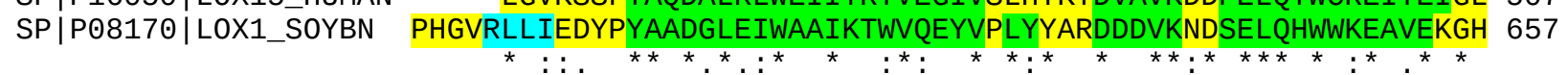

SP|P16050|LOX15_HUMAN QGAQDRGFPVSLQARDQVCHFVTMCIFTCTGQHASVHLGQLDWYSWVPNAPCTMRLPPPT 567 SP|P08170|LOX1_SOYBN GDLKDKPWWPKLQTLEDLVEVCLIIIWIASALHAAVNFGQYPYGGLIMNRPTASRRLLPE 717

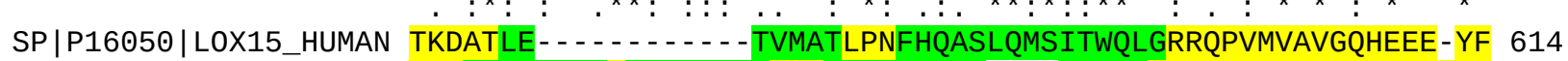
SP|P08170|LOX1_SOYBN KGTPEYEEMINNHEKAYLRTITSKLPTLISLS- - -VIEILSTHASDEVYLGQRDNPHWT 773 SP|P16050|LOX15_HUMAN SGPEPKAVLKKFREELAALDKEIEIRN - _ - _ - - AKLDMPYEYLRPSVVENSV - - - 660 SP|P๑8170|LOX1_SOYBN SDSKALQAFQKFGNKLKEIEEKLVRRNNDPSLQGNRLGPVQLPYTLLYPSSEEGLTFRGI 833

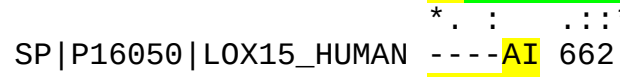
SP|PO8170|LOX1_SOYBN PNSISI 839 


\section{CONCLUSION}

Soybean LOX-1 might not be accepted as a model enzyme for 15-LOX from other sources as mentioned by several researchers that soybean LOX-1 used as a model enzyme for 15 LOX (human) $(14,16-18,52)$ since there are remarkable sequence-based differences which are obtained by using bioinformatics tools between soybean LOX-1 and LOXs from other sources especially from human. The future works are strongly warranted by using newly developed bioinformatics tools to find the appropriate enzyme model for human LOX. At this point, various genetic methods can be introduced and developed. The relevant enzymes can be produced in large scale by micro-organisms with the help of recombinant DNA technology for instance. Recently, the use of recombinant human lipoxygenase enzymes has become increasingly common due to such sensitivities in lipoxygenase inhibition studies $(35,48,53,54)$.

\section{ACKNOWLEDGMENTS}

We thank Prof. Dr. Theodore Holman from Chemistry and Biochemistry Department of UC Santa Cruz for sharing his valuable comments about LOXs.

\section{REFERENCES}

1. Jacquot C, McGinley CM, Plata E, Holman TR, van der Donk WA. Synthesis of 11-thialinoleic acid and 14-thialinoleic acid, inhibitors of soybean and human lipoxygenases. Organic and Biomolecular Chemistry. 2008 Dec;6(22):4242-52.

2. Newcomer ME, Brash AR. The structural basis for specificity in lipoxygenase catalysis. Protein Science. 2015 Mar;24(3):298-309.

3. Skrzypczak-Jankun E, McCabe NP, Selman SH, Jankun J. Curcumin inhibits lipoxygenase by binding to its central cavity: theoretical and X-ray evidence. International Journal of Molecular Medicine. 2000 Nov;6(5):521-6.

4. Simona V, Chedea VS, Dulf F, Socaciu C. The influence of quercetin concentration on its intermolecular interaction with soybean lipoxygenase-1. Bulletin USAMV-CN. 2006;62:37680.

5. Shrivastava SK, Srivastava $P$, Bandresh R, Tripathi PN, Tripathi A. Design, synthesis, and biological evaluation of some novel indolizine derivatives as dual cyclooxygenase and lipoxygenase inhibitor for anti-inflammatory activity. Bioorganic and Medicinal Chemistry. June 2017;25(16):4424-32.

6. Yashaswini PS, Appu Rao AG, Singh SA. Inhibition of lipoxygenase by sesamol corroborates its potential anti-inflammatory activity. International Journal of Biological Macromolecules 2017 Jan;94(Pt B):781-7.
7. ElBordiny HS, El-Miligy MM, Kassab SE, Daabees $\mathrm{H}$, Mohamed Ali WA, El-Hawash SAM. Design, synthesis, biological evaluation and docking studies of new 3-(4,5-dihydro-1H-pyrazol/isoxazol-5-yl)-2phenyl- $1 \mathrm{H}$-indole derivatives as potent antioxidants and 15-lipoxygenase inhibitors. European Journal of Medicinal Chemistry. 2018 Feb;145:594-605.

8. Boyington JC, Gaffney BJ, Amzel LM. The threedimensional structure of an arachidonic acid 15lipoxygenase. Science. 1993 Jun;260(5113):1482-6.

9. Ivanov I, Heydeck D, Hofheinz K, Roffeis J, O'Donnell VB, Kuhn $H$, Walther M. Molecular enzymology of lipoxygenases. Archives of Biochemistry and Biophysics. 2010 Nov;503(2):16174.

10. Segraves EN, Holman TR. Kinetic Investigations of the Rate-Limiting Step in Human 12- and 15Lipoxygenase. Biochemistry. 2003 May;42(18):523643.

11. Neidig ML, Wecksler AT, Schenk G, Holman TR, Solomon El. Kinetic and Spectroscopic Studies of N694C Lipoxygenase: A Probe of the Substrate Activation Mechanism of a Nonheme Ferric Enzyme. Journal of the American Chemical Society. 2007 Jun;129(24):7531-7.

12. Gardner HW. Recent investigations into the lipoxygenase pathway of plants. Biochimica et Biophysica Acta (BBA) - Lipids and Lipid Metabolism. 1991 Jul;1084(3):221-39.

13. Yamamoto S. Mammalian lipoxygenases: molecular structures and functions. Biochimica et Biophysica Acta (BBA) - Lipids and Lipid Metabolism. 1992 Oct;1128(2-3):117-31.

14. Maiga A, Malterud KE, Diallo D, Paulsen BS. Antioxidant and 15-lipoxygenase inhibitory activities of the Malian medicinal plants Diospyros abyssinica (Hiern) F. White (Ebenaceae), Lannea velutina A. Rich (Anacardiaceae) and Crossopteryx febrifuga (Afzel) Benth. (Rubiaceae). Journal of Ethnopharmacology. 2006 Mar;104(1-2):132-7.

15. Kuhn $H$, Walther $M$, Kuban RJ. Mammalian arachidonate 15-lipoxygenases structure, function, and biological implications. Prostaglandins and Other Lipid Mediators. 2002 Aug;68-69:263-90.

16. Mahesha HG, Singh SA, Appu Rao AG. Inhibition of lipoxygenase by soy isoflavones: Evidence of isoflavones as redox inhibitors. Archives of Biochemistry and Biophysics. 2007 May;461(2):17685.

17. Russell WR, Scobbie L, Duthie GG, Chesson A. Inhibition of 15-lipoxygenase-catalysed oxygenation of arachidonic acid by substituted benzoic acids. Bioorganic and Medicinal Chemistry. 2008 Apr;16(8):4589-93. 
18. Nguyen MD, Nguyen DH, Yoo JM, Myung PK, Kim MR, Sok DE. Effect of endocannabinoids on soybean lipoxygenase-1 activity. Bioorganic Chemistry. 2013 Aug;49:24-32.

19. Oso BJ, Karigidi KO. Inhibitory action of dried leaf of Cassia alata (Linn.) Roxb against lipoxygenase activity and nitric oxide generation. Scientia Agropecuaria. 2019 Mar;10(2):185-90.

20. Jonsson $T$, Glickman $M H$, Sun S, Klinman JP. Experimental Evidence for Extensive Tunneling of Hydrogen in the Lipoxygenase Reaction: Implications for Enzyme Catalysis. Journal of the American Chemical Society. 1996 Oct; 118(42):10319-20.

21. Gousiadou C, Kouskoumvekaki I. LOX1 inhibition with small molecules. Journal of Molecular Graphics and Modelling. 2016 Jan;63:99-109.

22. Sigal E, Craik CS, Highland E, Grunberger D, Costello LL, Dixon RA, Nadel JA. Molecular cloning and primary structure of human 15-lipoxygenase. Biochemical and Biophysical Research Communications. 1988 Dec;157(2):457-64.

23. Holman TR, Zhou J, Solomon El. Spectroscopic and Functional Characterization of a Ligand Coordination Mutant of Soybean Lipoxygenase-1: First Coordination Sphere Analogue of Human 15Lipoxygenase. Journal of the American Chemical Society. 1998 Nov;120(48):12564-72.

24. Minor W, Steczko J, Stec B, Otwinowski Z, Bolin JT, Walter R, Axelrod B. Crystal structure of soybean lipoxygenase $\mathrm{L}-1$ at $1.4 \mathrm{~A}$ resolution. Biochemistry. 1996 Aug;35(33):10687-701.

25. van Leyen $K$, Duvoisin RM, Engelhardt $H$, Wiedmann $M$. A function for lipoxygenase in programmed organelle degradation. Nature. 1998 Sep;395(6700):392-5.

26. Kuhn $H$, Thiele BJ. The diversity of the lipoxygenase family. Many sequence data but little information on biological significance. FEBS Letters. 1999 Apr;449(1):7-11.

27. Honn KV, Tang DG, Gao X, Butovich IA, Liu B, Timar J, Hagmann W. 12-lipoxygenases and 12(S)HETE: role in cancer metastasis. Cancer and Metastasis Reviews. 1994 Dec;13(3-4):365-96.

28. Werz O. Inhibition of 5-lipoxygenase product synthesis by natural compounds of plant origin. Planta Medica. 2007 Oct;73(13):1331-57.

29. Mhater $M$. The role of the 5-lipoxygenase pathway in Alzheimer's disease. Drugs of the Future. 2006 Jan;31(1):83-9.

30. Praticò D, Zhukareva V, Yao Y, Uryu K, Funk CD, Lawson JA, Trojanowski JQ, Lee VMY. 12/15Lipoxygenase is increased in Alzheimer's disease Possible involvement in brain oxidative stress.
American Journal Of Pathology. 2004 May;164(5):1655-62.

31. Schenk G, Neidig ML, Zhou J, Holman TR, Solomon El. Spectroscopic Characterization of Soybean Lipoxygenase-1 Mutants: the Role of Second Coordination Sphere Residues in the Regulation of Enzyme Activity. Biochemistry. 2003 Jun;42(24):7294-302.

32. Cengiz S, Cavas L, Yurdakoc K, Pohnert G. The sesquiterpene caulerpenyne from Caulerpa spp. is a lipoxygenase inhibitor. Marine Biotechnology. 2011 Apr;13(2):321-6.

33. Richter $P$, Schubert G, Schaible AM, Cavas L, Werz O, Pohnert G. Caulerpenyne and Related Bisenol Esters Are Novel-Type Inhibitors of Human 5Lipoxygenase. ChemMedChem. 2014 Aug;9(8):16559.

34. Omar YM, Abdu-Allah HHM, Abdel-Moty SG. Synthesis, biological evaluation and docking study of 1,3,4-thiadiazolethiazolidinone hybrids as antiinflammatory agents with dual inhibition of COX-2 and 15-LOX. Bioorganic Chemistry. 2018 Oct;80:461-71.

35. van der Vlag R, Guo H, Hapko U, Eleftheriadis N, Monjas L, Dekker FJ, Hirsch AKH. A combinatorial approach for the discovery of drug-like inhibitors of 15-lipoxygenase-1. European Journal of Medicinal Chemistry. 2019 Jul;174:45-55.

36. Bouriche $H$, Miles EA, Selloum L, Calder PC. Effect of Cleome arabica leaf extract, rutin and quercetin on soybean lipoxygenase activity and on generation of inflammatory eicosanoids by human neutrophils. Prostaglandins Leukotrienes and Essential Fatty Acids. 2005 Mar;72(3):195-201.

37. Zhang YY, Thakur K, Wei CK, Wang H, Zhang JG, Wei ZJ. Evaluation of inhibitory activity of natural plant polyphenols on Soybean lipoxygenase by UFLC-mass spectrometry. South African Journal of Botany. 2019 Jan;120:179-85.

38. Sievers F, Wilm A, Dineen DG, Gibson TJ, Karplus K, Li W, Lopez R, McWilliam H, Remmert M, Söding J, Thompson JD, Higgins DG. Fast, scalable generation of high-quality protein multiple sequence alignments using Clustal Omega. Molecular Systems Biology. 2011 Oct; 7:539.

39. Madeira F, Park YM, Lee J, Buso N, Gur T, Madhusoodanan N, Basutkar P, Tivey ARN, Potter SC, Finn RD, Lopez R. The EMBL-EBI search and sequence analysis tools APIs in 2019. Nucleic Acids Research. 2019 Jul;47(W1):W636-W641.

40. Gasteiger E, Hoogland C, Gattiker A, Duvaud S, Wilkins MR, Appel RD, Bairoch A. Protein Identification and Analysis Tools on the ExPASy Server. In: Walker JM (eds) The Proteomics Protocols Handbook. Humana Press, 2005;pp 571-607. 
41. Gasteiger E, Gattiker A, Hoogland C, Ivanyi I, Appel RD, Bairoch A. ExPASy: the proteomics server for in-depth protein knowledge and analysis. Nucleic Acids Research. 2003 Jul;31(13):3784-8.

42. Jones DT. Protein secondary structure prediction based on position-specific scoring matrices. Journal of Molecular Biology. 1999 Sep;292(2):195-202.

43. Kyte J, Doolittle RF. A simple method for displaying the hydropathic character of a protein. Journal of Molecular Biology. 1982 May;157(1):10532.

44. Mogul R, Holman TR. Inhibition Studies of Soybean and Human 15-Lipoxygenases with LongChain Alkenyl Sulfate Substrates. Biochemistry. 2001 Apr;40(14):4391-7.

45. Galliard T, Chan HWS. Lipoxygenases. In: Stumpf PK, Conn EE (eds) The Biochemistry of Plants. Academic Press, New York, 1980;pp 4:131-61.

46. Vidal-Mas J, Busquets $M$, Manresa A. Cloning and expression of a lipoxygenase from Pseudomonas aeruginosa 42A2. Antonie Van Leeuwenhoek. 2005 Apr;87(3):245-51.

47. Sadeghian $H$, Jabbari A. 15-Lipoxygenase inhibitors: a patent review. Expert Opinion on Therapeutic Patents. 2016;26(1):65-88.

48. Amagata $T$, Whitman $S$, Johnson TA, Stessman CC, Loo CP, Lobkovsky E, Clardy J, Crews P, Holman TR. Exploring Sponge-Derived Terpenoids for Their Potency and Selectivity against 12-Human, 15-
Human, and 15-Soybean Lipoxygenases. Journal of Natural Products. 2003 Feb;66(2):230-5.

49. Prigge ST, Boyington JC, Gaffney BJ, Amzel LM. Structure Conservation in Lipoxygenases: Structural Analysis of Soybean Lipoxygenase-1 and Modeling of Human Lipoxygenases. Proteins Structure Function and Bioinformatics. 1996 Mar;24(3):275-91.

50. Gillmor SA, Villasenor A, Fletterick R, Sigal E, Browner MF. The structure of mammalian 15lipoxygenase reveals similarity to the lipases and the determinants of substrate specificity. Nature Structural Biology. 1997 Dec;4(12):1003-9.

51. Choi J, Chon JK, Kim S, Shin W. Conformational flexibility in mammalian 15S- lipoxygenase: Reinterpretation of the crystallographic data. Proteins. 2008 Feb;70(3):1023-32.

52. Serpen A, Gökmen V. Effects of $\beta$-carotene on soybean lipoxygenase activity: kinetic studies. European Food Research and Technology. 2007 Apr;224(6):743-8.

53. Eleftheriadis $\mathrm{N}$, Thee $\mathrm{S}$, te Biesebeek J, van der Wouden P, Baas BJ, Dekker FJ. Identification of 6benzyloxysalicylates as a novel class of inhibitors of 15-lipoxygenase-1. European Journal of Medicinal Chemistry. 2015 Apr;94:265-75.

54. Guo $H$, Verhoek IC, Prins GGH, van der Vlag $R$, van der Wouden PE, van Merkerk R, Quax WJ, Olinga $P$, Hirsch AKH, Dekker FJ. Novel 15-Lipoxygenase-1 Inhibitor Protects Macrophages from Lipopolysaccharide-Induced Cytotoxicity. Journal of Medicinal Chemistry. 2019 May;62(9):4624-37. 
